\title{
The Foundations of Human Morality: A Critical Analysis of Moral Nativism
}

by

\author{
Nalini Elisa Ramlakhan
}

A thesis submitted to the Faculty of Graduate and Postdoctoral Affairs

in partial fulfillment of the requirements for the degree of

Master of Arts

in

Philosophy

Carleton University
Ottawa, Ontario

(C) 2011

Nalini Elisa Ramlakhan 
Library and Archives

Canada

Published Heritage

Branch

395 Wellington Street

Ottawa ON K1A ON4

Canada
Bibliothèque et

Archives Canada

Direction du

Patrimoine de l'édition

395 , rue Wellington

Ottawa ON K1A ON4

Canada
Your file Votre référence

ISBN: 978-0-494-87787-6

Our file Notre référence

ISBN: $978-0-494-87787-6$

\section{NOTICE:}

The author has granted a nonexclusive license allowing Library and Archives Canada to reproduce, publish, archive, preserve, conserve, communicate to the public by telecommunication or on the Internet, loan, distrbute and sell theses worldwide, for commercial or noncommercial purposes, in microform, paper, electronic and/or any other formats.

The author retains copyright ownership and moral rights in this thesis. Neither the thesis nor substantial extracts from it may be printed or otherwise reproduced without the author's permission.
AVIS:

L'auteur a accordé une licence non exclusive permettant à la Bibliothèque et Archives Canada de reproduire, publier, archiver, sauvegarder, conserver, transmettre au public par télécommunication ou par l'Internet, prêter, distribuer et vendre des thèses partout dans le monde, à des fins commerciales ou autres, sur support microforme, papier, électronique et/ou autres formats.

L'auteur conserve la propriété du droit d'auteur et des droits moraux qui protege cette thèse. $\mathrm{Ni}$ la thèse ni des extraits substantiels de celle-ci ne doivent être imprimés ou autrement reproduits sans son autorisation.
In compliance with the Canadian Privacy Act some supporting forms may have been removed from this thesis.

While these forms may be included in the document page count, their removal does not represent any loss of content from the thesis.
Conformément à la loi canadienne sur la protection de la vie privée, quelques formulaires secondaires ont été enlevés de cette thèse.

Bien que ces formulaires aient inclus dans la pagination, il n'y aura aucun contenu manquant. 


\begin{abstract}
The aim of this paper is to determine the plausibility of moral nativism by examining and analyzing three main components of moral nativism, the poverty of moral stimulus argument, which is the first part of the Linguistic Analogy, moral dumbfounding, which is intertwined with, but separate from, the Linguistic Analogy, and the second part of the Linguistic Analogy, the Principles and Parameters approach. After analyzing the arguments in favour of moral nativism, I argue that these three components of moral nativism are problematic and lack support. I argue that if moral acquisition can be adequately explained by a nonnativist account, then there are grounds for rejecting both moral nativism and the proposal that there are universal moral domains that guide moral decision-making. This paper aims to point out the problems associated with moral nativism that many nativists overlook, do not address in their work, or do not consider problematic for their position, and argues that the inability to prove that nonnativist theories are inadequate or insufficient in accounting for moral acquisition and moral decisionmaking weakens the nativist position. In an attempt to dismiss moral nativism as implausible, I provide three alternative nonnativist accounts of moral decision-making that plausibly account for moral acquisition and variation in moral decision-making across cultures and within cultures, as well as the emergence, maintenance, and transmission of moral norms.
\end{abstract}




\section{Acknowledgements}

I would like to begin by thanking Dr. Andrew Brook for supervising my thesis and for all of his support. Having Dr. Brook as a supervisor and working with him on this project has been a delightful experience, and I am grateful to Dr. Brook for all of his advice, comments, and time that was put into this project. Many thanks, Dr. Brook.

I would also like to thank Dr. Heidi Maibom, and Dr. Christopher Burn for agreeing to be on my defense committee.

I would especially like to thank Dr. David Matheson for all of his insightful comments, support, and guidance throughout the duration of this project.

Lastly, I would like to thank my parents who have been supportive and encouraging throughout the entirety of this project. Thank you for always pushing me to do the best that I can and for supporting me throughout this project. Without your love and support, this project would not have been possible. 


\section{Contents}

Abstract.......................................................................

Acknowledgements...........................................................

Introduction........................................................................

Chapter 1 - An Introduction to Moral Nativism.................................... 10

Chapter 2 - Problems with Moral Nativism.......................................49

Chapter 3 - Alternatives to Moral Nativism.........................................85

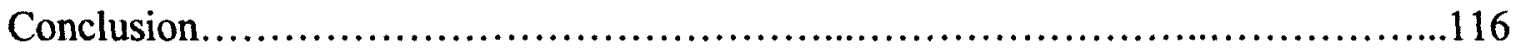

Bibliography............................................................... 118 


\section{Introduction}

Questions pertaining to the nature of moral judgments are long standing in the field of philosophy, and although philosophy has progressed significantly throughout the years, these questions remain debated among philosophers. In fact, new questions have been raised in an attempt to understand the nature of moral judgments. This paper is concerned with analyzing the following question: are humans innately equipped with moral principles that account for moral acquisition and moral decision-making, or is morality acquired solely through environmental factors? Those who favour the view that humans are equipped with moral principles that account for moral acquisition argue that certain moral principles are innate, and cultures vary in moral decision-making depending on how the social environment shapes these principles. This view is known as the theory of moral nativism, where nativism is the term used for an innate endowment. Those arguing in favour of moral nativism tend to liken innate moral principles to innate language principles, where universal grammar is innate, but which language we speak is dependent upon which society we are raised in. These arguments take the position of Rawls's Linguistic Analogy, where he argued in A Theory of Justice (1971) that we have a moral grammar that is analogous to Chomsky's Principles and Parameters theory in language. Those arguing in favour of the view that moral acquisition is acquired through sociocultural and environmental factors hold a nonnativist view of morality. Proponents of this theory do not contest the fact that humans have a capacity to act in a moral manner or to think morally. Rather, they take issue with the claim that a moral faculty or a universal moral grammar is needed to explain moral acquisition.

It is important to note that nativism is simply another term for innatism. While it seems more commonsensical to use the term innatism when speaking of an innate endowment, 
nativism, at least in the literature of moral psychology, has become the common term for innatism. Thus, I use the term nativism throughout this paper to refer to innatism, and use the terms interchangeably.

There are a significant number of scholars who have contributed, and continue to contribute, to this area in moral psychology. Biologist Marc Hauser, philosopher Susan Dwyer, and psychologist Jonathan Haidt are moral nativists who provide stimulating and persuasive arguments in favour of moral nativism. Philosophers Jesse Prinz, Chandra Sripada, and Daniel Dennett $^{1}$ are advocates of nonnativist theories of morality, and provide accounts of moral acquisition that rely heavily on sociocultural and environmental factors. While there are many scholars who have contributed to this debate, this paper will focus mainly on the works of the scholars mentioned.

The aim of this paper is to determine the plausibility of moral nativism by examining and analyzing three main components of moral nativism, the poverty of moral stimulus argument, which is the first part of the Linguistic Analogy, moral dumbfounding, which is intertwined with, but separate from, the Linguistic Analogy, and the second part of the Linguistic Analogy (LA hereafter), the Principles and Parameters approach. After analyzing the arguments in favour of moral nativism, I argue that these three components of moral nativism are problematic and lack support. I argue that if moral acquisition can be adequately explained by a nonnativist account, then there are grounds for rejecting both moral nativism and the proposal that there are universal moral domains that guide moral decision-making. This paper aims to point out the problems associated with moral nativism that many nativists overlook, do not address in their work, or do not consider problematic for their position, and argues that the inability to prove that nonnativist theories are inadequate or insufficient in accounting for moral acquisition and moral decision- 
making weakens the nativist position. In an attempt to dismiss moral nativism as implausible, I provide three alternative nonnativist accounts of moral decision-making that plausibly account for moral acquisition and variation in moral decision-making across cultures and within cultures, as well as the emergence, maintenance, and transmission of moral norms.

In order to defend the claim that moral nativism is problematic, I begin Chapter I with an explication of moral nativist theories, where I provide a detailed account of what moral nativism consists of and introduce the theories put forth by Hauser, Dwyer, and Haidt. In particular, the poverty of moral stimulus argument, moral dumbfounding, and the Principles and Parameters theory will be explicated at length. In doing so, we will look at hypothetical moral dilemmas that are used by moral nativists to support their theories.

In Chapter II, we turn to the problems associated with moral nativist theories. In this chapter, I critically examine the similar moral nativist theories presented by Hauser and Dwyer, followed by a critical examination of Haidt's Social Intuitionist Approach to Morality (SIM hereafter $)^{2}$ which is a distinct theory from Hauser and Dwyer's theories. In this chapter, we will look at three particular issues associated with moral nativism: first, issues with the poverty of moral stimulus argument, where I argue that the environment may be too impoverished to supply children with language but this is not the case for moral behaviour, in addition to examining why it is implausible to suggest that moral dumbfounding is a real phenomenon; second, the problems associated with using the LA with regard to Principles and Parameters, where we will examine why it is plausible to claim that humans are endowed with a language faculty but not a moral faculty; and third, the claim made by many philosophers that universality establishes innateness. In addition, we will examine the issues embedded in Haidt's SIM and look at the similarities between Haidt's SIM and nonnativist theories. 
In the third and final chapter, we turn to nonnativist accounts of morality and determine if all, or any, of these theories can account for moral acquisition. In analyzing these theories, I argue that each theory plausibly accounts for moral acquisition and does so without postulating a moral faculty. In examining and analyzing nonnativist accounts of morality, I briefly consider evolutionary constraints on social and moral behaviour and conclude that all things considered, speculations about evolution and morality lend support to a nonnativist account of morality, despite the evolutionary arguments that nativists provide.

\section{Scope and Limits}

This project opens the door for many questions, such as what are the implications of a nativist or nonnativist view in ethical theory? Does a nonnativist view lead to ethical naturalism or relativism about morality? Unfortunately, the scope of this project does not allow me to address or examine these questions, despite their importance. This paper focuses solely on analyzing and evaluating the plausibility of moral nativism, looking at the difficulties associated with moral nativism, and attempting to provide plausible alternatives to moral nativist theories.

\section{Preliminary Clarifications}

Before we begin our discussion of moral nativism, we must start with a few clarifications and definitions. Generally speaking, in terms of the relevant context, nativists believe that a specific aspect or feature of human cognition, whether it is language, morality, a concept of God, etc., is innate in the human brain or mind. Whether that aspect was stamped on the human mind by a supernatural being, as Descartes postulated when determining how humans come to know and believe in God, or whether there are evolutionary considerations for the innate endowment, which is the view of most contemporary nativists, is beside the point. Nativists hold that because 
we display certain features in our psychology that cannot be explained solely through environmental factors, the features must be innate.

In the beginning of this introduction, I briefly defined moral nativism and nonnativism, so I will not provide definitions of these terms here. However, before delving into our discussion of moral nativism, we need to distinguish between two different kinds of moral nativism: capacity nativism and content nativism. Capacity nativism entails that an individual has certain cognitive capacities, such as the capacity to attribute mental states to other individuals (a theory of mind or simulation) or the capacity to distinguish between intended and accidental actions (a theory of action). Capacity nativism is concerned with which cognitive capacities may be needed to make a moral judgment and which may be involved in moral decision-making, and these questions may receive a variety of answers. However, what is not contested is the claim that all humans have the capacity to act in a moral manner, to make moral decisions, or simply, to moralize. It is important to note that nonnativists are not contesting the claim that humans have a capacity to moralize, thus nonnativists about morality do not reject capacity nativism.

Nonnativists take issue with content nativism. Content nativism is concerned with whether there are innate structures in the human mind that shape the content of moral norms, where "the content of a moral norm consists of the class of actions that the norm prohibits, permits, or requires. ${ }^{3}$ Usually when moral nativists argue in favour of moral nativism, they are arguing for content nativism, namely, the view that there are innate structures in the brain that shape the content of moral norms. These innate structures consist of principles, such as a principle of harm or purity, that guide moral decision-making. In addition, a moral principle can include not only principles such as harm, fairness, etc., but also the ability to distinguish between moral and conventional norms, or the ability to distinguish between intended and accidental 
consequences and calibrating the level of punishment accordingly. According to moral nativism, moral principles can also include doctrines, such as the doctrine of double effect. How many principles and which principles are included in the moral faculty are questions that receive different answers from different theorists.

Moreover, when speaking in terms of a moral faculty, some moral nativists postulate that the moral faculty is a Neocartesian kind of mechanism. A Neocartesian module or mechanism is similar to Fodor's conception of vertical faculties. Fodorian modules operate automatically and quickly, where the unconscious principles that guide output cannot be accessed. A Fodorian module is innate and encapsulated, where the module is isolated from other parts of cognition, and "the outputs of modules are shallow in the sense of being nonconceptual...(Fodorian) modules generate information of various sorts, but they don't issue in thoughts or beliefs.", Neocartesian faculties focus on the propositional structure of the innate knowledge being represented, rather than the psychological mechanisms that are needed to put these principles to work. What is innate in a Neocartesian mechanism is a body of information that constitutes propositional attitudes. A worry for proponents of this view is that a Neocartesian faculty leaves open which mechanisms are needed to ensure that the principles are at work. Moreover, there must be more to how the mind works than propositional content alone. ${ }^{5}$ In terms of the relevant context, Neocartesian faculties are similar to vertical faculties with the exception of a mechanism that ensures that innate principles are at work. ${ }^{6}$ Dwyer maintains that the moral faculty can be thought of as a Neocartesian faculty, and we will explore this view in Chapter I.

Other nativists, such as Haidt, maintain that moral principles take the form of a Sperberian module. ${ }^{7}$ A Sperberian module is the mediate position between an extreme view of modularity (Fodor's conception of modularity or massive modularity as proposed by Pinker and 
Cosmides and Tooby) and the view that the mind is prepared by evolution (e.g., there is a minimal amount of structure on the human mind at birth due to the fact that the human brain has been shaped by evolution, and this allows us to acquire certain kinds of knowledge, like moral knowledge). Sperberian modules do not need to be as tightly modularized as Fodorian modules in terms of higher cognition. Rather, "higher cognitive processes are modularized 'to some interesting degree,' that is, higher cognition is not one big domain-general cognitive work space. ${ }^{\prime 8}$ On this view, morality can be viewed as a set of multiple concerns about social life, where each concern has an evolutionary story and psychological mechanism, where the mechanism requires guidance from culture and the environment to develop properly. ${ }^{9}$ We will explore this view in more detail in the following chapter. What is important to note in the meantime is that while there is disagreement among moral nativists in terms of what kind of mechanism the moral faculty is, moral nativists are primarily concerned with defending some form of content nativism.

It is important to note that when speaking of moral principles, I use the term broadly to include both innate principles and innate moral domains or foundations. Haidt does not argue in favour of moral principles. Rather, he argues in favour of innate foundations or domains of moral decision-making. To briefly elaborate, Haidt believes that moral principles take the form of innate moral foundations, where a foundation can be viewed as a Sperberian module. According to Haidt, there are five foundations or domains of moral decision-making: harm, purity, fairness, hierarchy, and ingroup. These five foundations form the basis of moral decision-making, and humans would be unable to moralize without them. Haidt's theory shares many commonalities with both Hauser and Dwyer's theories, and innate moral domains are very similar to innate 
moral principles as we will see in the following chapter, therefore I use the term moral principles to include Haidt's five foundations of morality.

We must also distinguish between universality and innateness, as the two terms tend to be used interchangeably, and many assume that universality implies innateness. We have already defined innateness above, so we can skip right to defining universality. In terms of our context, universality simply means that a feature of humans, in this case human morality, is present and common across individuals, irrespective of location. To say that something is universal in humans is to say that it is a shared feature of being human. For example, having hands is a universal feature of being human. Nearly all humans, irrespective of location, are born with hands. In the case of hands, we can say that having hands is both a universal and innate feature of being human. It is universal because we all have hands and are all born with hands, and it is innate because there are genes that regulate the growth of hands. Thus, hands are an innate endowment and a universal feature of being human.

The final point of clarification before moving into our discussion concerns whether all nativist theories postulate moral principles. The question that must be asked is the following: can there be a nativist theory that does not propose innate moral principles? Simply, the answer is no. There are two reasons for this: first, I have yet to come across any serious account of moral nativism that does not postulate moral principles of some sort, and second, postulating a moral faculty without moral principles, namely the innate structures in the mind that shape moral decision-making, would be to postulate something along the lines of capacity nativism, which everyone, even a hardnosed empiricist, agrees with. Thus, when I speak of moral nativism, I refer to the theories that endorse the view that the moral faculty is composed of specific innate principles, such as a principle of harm, that guide moral decision-making. 
It is also important to keep in mind that moral nativism comes in a variety of flavours. By this I mean that moral nativism can range from extreme, to moderate, to mild in terms of what the doctrine claims to be innate. For instance, a moral nativist doctrine may claim that everything about morality is innate in humans, where the environment plays absolutely no role. Or a nativist theory, such as Hauser's, may be a moderate to strong theory and claim that certain principles of morality, such as the doctrine of double effect, are innate, and the environment plays a role in shaping the principles. The three theories to be explicated in the following chapter advocate a moderate to strong theory of moral nativism, but we must not forget that moral nativism ranges in its extremity depending on the theory.

Notes

\footnotetext{
${ }^{1}$ I focus mostly on Prinz and Sripada's theories of nonnativism, as Dennett does not wholly advocate a nonnativist account of morality. Rather, I include parts of Dennett's theory to make a strong case for nonnativism.

${ }^{2}$ Jonathan Haidt and Fredrik Bjorklund, "Social Intuitionists Answer Six Questions about Moral Psychology" as an article in Walter Sinnott-Armstrong, Moral Psychology: The Cognitive Science of Morality: Intuition and Diversity vol. 2 (Cambridge: MIT Press, 2008), 181.

${ }^{3}$ Chandra Sekhar Sripada, "Nativism and Moral Psychology: Three Models of the Innate Structure That Shapes the Contents of Moral Norms" as an article in Walter Sinnott-Armstrong, Moral Psychology: The Evolution of Morality: Adaptations and Innateness vol. 1 (Cambridge: MIT Press, 2008), 321.

${ }^{4}$ Peter Carruthers, The Architecture of the Mind (New York: Oxford University Press, 2006), 5, parentheses mine.

${ }^{5}$ Jerry A. Fodor, The Modularity of Mind (Cambridge: MIT Press, 1983), 9.

${ }^{6}$ There are many differences between a Fodorian module and a Neocartesian mechanism that do not concern our discussion. See Fodor 1983 for a lengthy discussion on the differences between the mechanisms.

${ }^{7}$ For more on modules and the different kind of modules modularity theorists put forth, see Peter Carruthers, "The Case for Massively Modular Models of Mind" as an article in Robert J. Stainton, Contemporary Debates in Cognitive Science (Malden: Blackwell publishing, 2006).

${ }^{8}$ Jonathan Haidt and Fredrik Bjorklund, "Social Intuitionists Answer Six Questions about Moral Psychology" as an article in Walter Sinnott-Armstrong, Moral Psychology: The Cognitive Science of Morality: Intuition and Diversity vol. 2 (Cambridge: MIT Press, 2008), 205.

${ }^{9}$ Haidt and Bjorklund, 206.
} 


\section{Chapter I: An Introduction of Moral Nativism and Moral Nativists}

In the Introduction I provided a definition of moral nativism and briefly introduced three proponents of moral nativism. In this chapter, I look at moral nativism and examine the theories proposed by Marc Hauser, Susan Dwyer, and Jonathan Haidt, while touching on work by other moral nativists, such as Steven Pinker and John Mikhail. Rather than focusing on each theory separately, I focus on the commonalities of the theories, especially the commonalities that Hauser and Dwyer's work share, in order to better situate the argument in favour of moral nativism. The first component of moral nativism I discuss is the first part of the LA, the poverty of moral stimulus argument, where I explicate the arguments in favour of the view that the environment cannot supply children with sufficient stimuli for moral acquisition. Following this, I look at moral dumbfounding, a phenomenon that is separate from, but intertwined with, the LA. Then, I look at the second component of the Linguistic Analogy (LA), Principles and Parameters, and arguments from Dwyer and Hauser that suggest moral acquisition is similar to language acquisition. Finally, I look at arguments suggesting that there is universality in moral decision-making which entails that humans are endowed with an innate moral faculty. In doing so, I look at Haidt's Social Intuitionist Approach to Morality (SIM) and his conception of the five foundations or domains of moral decision-making, where he argues that these five domains guide moral decision-making and inevitably lead to a particular way of making a moral judgment.

What is moral nativism? Moral nativism is the view that all humans, irrespective of location, are endowed with innate moral principles that guide moral-decision making. Because of these innate principles, there is universality in moral-decision making despite cross-cultural differences. Although we witness differences across cultures, moral nativists claim that this is 
because environmental factors play a significant role in shaping morality; although there are differences across cultures in moral decision-making, at a general level there is universality. For example, moral nativists claim that all individuals have an innate principle prohibiting harm. This is why every culture has some form of a harm norm, although harm norms differ across cultures (e.g., some honour cultures murder their partners for adultery while others find this morally reprehensible). Therefore, the principle of harm is innate, but how it is shaped and which harm norms an individual accepts is dependent upon which environment the individual is raised in. Although individuals and cultures do not agree on the specific content of harm norms, harm norms are a universal feature of the moral faculty. What is more, in specific moral dilemmas, moral nativists claim that individuals respond in a universal manner, and this is because of our moral faculty. How many moral principles humans are endowed with and what these principles are, are two questions that are answered differently among nativists. However, most moral nativists agree that humans are endowed with an innate moral principle of harm, where humans can distinguish between intended and foreseen consequences, and will usually permit necessary harm under highly specific circumstances (e.g., self-defense) while avoiding harm in most situations. The principle of harm guides moral decision-making by allowing individuals to permit harm if the consequences of permitting harm are not intended but possibly foreseen, but not if the consequences of permitting harm are intended. As psychologist and cognitive scientist Steven Pinker describes the principle of harm, we have a revulsion to "manhandling an innocent person.",

In order to support the view that humans are equipped with an innate harm principle, moral nativists tend to cite biologist Marc Hauser's 2007 web-based experiment, where Hauser and colleagues created an experiment that extended beyond the laboratory. In this experiment, 
Hauser and colleagues presented over five thousand subjects ${ }^{2}$ with many ethical dilemmas to determine if individuals from different ethnic, age, and gender groups would respond in a similar manner to the dilemmas. They found that in most dilemmas, individuals did respond in the same manner. Because of this universality in moral decision-making, they concluded that humans must have a moral organ, a universal moral grammar that allows individuals to think and react in similar manners when making moral judgments. ${ }^{3}$

A well-known and often-cited moral dilemma that moral nativists use in support of a moral faculty is the Trolley Dilemma. In this dilemma, individuals are posed with the following situation: there is an out-of-control trolley heading in the direction of five hikers. The trolley is unable to stop, and will result in killing the five hikers who are unaware that the trolley is headed in their direction. On another path of the track, there is one individual who will not be hit and killed by the trolley if it continues on the path it is on. The question is whether it is morally right to pull a lever that will redirect the trolley on the path where the single individual resides, thus killing him and saving the lives of the five hikers. Most people claim that pulling the lever is a morally right action. When posed with a slightly different version of this dilemma, where the only way to save the five hikers is to physically push a large man off a bridge because he is the only object heavy enough to stop the trolley from running over and killing the five hikers, most people respond that this would be a morally wrong action. When Hauser and colleagues posed this question to subjects in their web-based experiment, they found that individuals responded in the same manner, irrespective of location.

In alternative trolley dilemmas posed by Hauser, where the dilemmas are slightly altered, Hauser and colleagues found that individuals responded in very similar manners to the dilemmas. We will explore these dilemmas in Chapter III, but it is important to keep in mind that on 
Hauser's account, individuals will respond in similar manners because of a moral faculty, and Hauser takes universality in moral decision-making to be evidence in favour of a moral organ.

In his stimulating book Moral Minds, Hauser claims that "we evolved a moral instinct, a capacity that naturally grows within each child, designed to generate rapid judgments about what is morally right or wrong based on an unconscious grammar of action." ${ }^{, 4}$ Part of this machinery that helps guide moral decision-making was designed by natural selection, millions of years prior to the evolution of our species, and other parts of the machinery were added or upgraded throughout human evolution. Hauser's ideas surrounding the possibility of a moral organ draw on insights from language, another human instinct in Hauser's view. ${ }^{5}$ He claims that in the same way that we have a universal grammar, namely, the ability to speak and comprehend a specific language without reasoning and without accessing the conscious rules or principles that allow for the proper use of grammar and semantic comprehension, we have a universal moral grammar. Our language faculty is a toolkit for building specific languages, such as Italian, Spanish, English, etc. Similarly, on Hauser's account, our moral faculty is a toolkit for building moral systems.

The language faculty consists of many rules and principles that are unconscious and inaccessible to us, and these principles allow us to comprehend and speak the dominant language in our society. We all have a language faculty that comes equipped with principles and the ability to learn any language, and depending on which society we are raised in, we will come to express the rules of the language that the society speaks unconsciously and with little help from environmental stimuli. Each individual comes equipped with principles that allow him/her to acquire language, and the environment that an individual is placed in determines which language will be their first. 
When linguists refer to the innate set of principles that are required for language acquisition, they are referring to the speaker's grammar, namely "the rules or operations that allow any normally developing human to unconsciously generate and comprehend a limitless range of well-formed sentences in their native language." ${ }^{\prime 6}$ When speaking about a universal grammar, linguists are referring to this point in particular. Similarly, because moral nativists compare morality to language, when referring to a universal moral grammar, moral nativists are referring to the set of principles required for moral acquisition, namely the rules or operations that allow a normally developing human to unconsciously generate and comprehend moral norms that are particular to their society. We will discuss the language faculty later on in this chapter when we look at the LA.

In an attempt to explain moral grammar, namely our toolkit that allows for the building of specific moral systems, Hauser claims that acquiring our culture's specific moral norms is a process that "is more like growing a limb than sitting in Sunday school and learning about vices and virtues--we judge whether actions are permissible, obligatory, or forbidden, without conscious reasoning and without explicit access to the underlying principles." ${ }^{, 7}$ Thus, this process is an unconscious process and like the principles of universal grammar, innate moral principles operate very quickly. Because moral decision-making is produced by an unconscious process, Hauser argues that when asked to justify our reasoning behind our judgements, we are often dumbfounded. ${ }^{8}$ In the same way that we are unable to express the principles that guide our language comprehension and use of grammar, we are unable to express the principles that guide our moral judgments. Dumbfounding is the phenomenon of making a judgment, such as incest is wrong even if there are no negative consequences, and then when asked for justification, 
individuals "stutter, laugh, and express surprise at their inability to find supporting reasons,"” yet they stick to their initial judgment.

Hauser gives many examples of moral dilemmas where we make a moral judgment but then experience dumbfounding. For instance, he claims that most of us agree that upon encountering an injured child, we have a moral obligation to assist the child despite the fact that helping the child will incur a cost of two hundred dollars. However, most of us agree that we do not have a moral obligation to donate fifty dollars to UNICEF in order to save the lives of twenty-five children. ${ }^{10}$ When pressed to explain why we are morally obligated to help the child with the injury, where helping will cost us two hundred dollars, but not morally obligated to help twenty-five children, where helping will cost us fifty dollars, we are dumbfounded as to why or how we reached this conclusion. On Hauser's model of a moral faculty, this does not entail that we do not reason when we make a moral judgment. Rather, the reasoning occurs unconsciously and a moral judgment is made after unconscious reasoning takes place. In addition, emotion may fuse with unconscious reasoning before making a moral judgment, but emotion is not a necessary factor on Hauser's account of a moral faculty. We will take another look at dumbfounding later on in the chapter.

In claiming that we are equipped with a moral faculty with inaccessible principles that guide moral decision-making, Hauser argues that children do not receive enough feedback from parents and educators to make a distinction between moral and conventional rules, and the ability to make this distinction is a product of the moral faculty. This is also known as the poverty of moral stimulus argument, the first part of the LA, which we will discuss later on in this chapter and consider as a separate argument in favour of a moral faculty. Hauser claims that a parent gets angry when their child does something wrong, such as when their child hits another child, and 
explains to the child that hitting is wrong, and the child is then able to read an emotion off their parent's face, such as anger or disappointment. The child is then able to map this expression onto previous events and understand that hitting is wrong. However, a parent may express the same or a similar emotion when the child begins eating sand from the sandbox and the child is able to

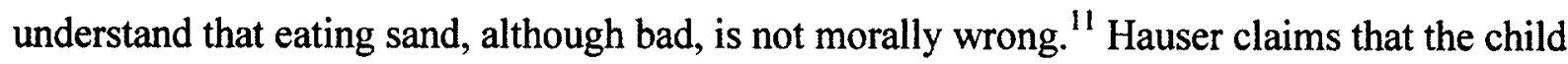
does not receive enough feedback in these situations to differentiate between a moral and conventional norm. Thus, being able to evaluate the difference between the two situations is a product of the moral faculty. We will return to distinguishing between moral and conventional norms in Chapters II and III.

Hauser also considers whether other mechanisms in the brain play a role in moral decision-making, and concludes that while other capacities, faculties, and mechanisms, such as a theory of mind faculty (or simulation) and working memory, play a role in moral decisionmaking, none of these other mechanisms are specific to moral decision-making, although they help with the process. In addition, and this is important to note as we will return to this point in Chapter III, Hauser claims that while emotions may sometimes play a role in moral decisionmaking, "our emotions are not part of the dedicated and specialized components of the moral faculty." 12 Rather, the moral faculty and its specialized components, that is, the components that are specific to the moral faculty rather than the shared components such as working memory, enables every normally developing child to acquire systems of morality. The system of morality that the child acquires is dependent upon the society that the child is raised in, in the same way that acquiring English as a first language requires that the child is raised in a society where English is the dominant language. In order to function properly and well, the moral faculty must interact with other mechanisms and capacities. 
Hauser claims that because of our moral organ, humans are really Rawlsian creatures at heart, rather than Kantian or Humean. Rawlsian creatures are described as creatures with moral instincts that are equipped with machinery to deliver moral judgments based on principles that are unconscious and cannot be made conscious. ${ }^{13}$ Kantian creatures perceive an event and deliver moral judgments "based on conscious reasoning from relevant principles." ${ }^{\prime 14}$ Humean creatures perceive an event and are guided by emotions when delivering a moral judgment. For Humean creatures, emotional responses generate moral judgments. It is important to take note of this as we will see later on in the chapter how nativist theories, although they share many commonalities, differ from one another. Haidt claims that because humans are endowed with innate moral modules, we tend to act as Humean creatures rather than Kantian or Rawlsian.

Hauser's theory of a moral faculty includes many more claims supported by persuasive arguments in favour of a moral organ. For example, he points out that individuals with damage to regions of the brain that are needed for moral decision-making are unable to make appropriate moral judgments. Hauser also draws on psychology studies in order to further support the theory of a moral organ. However, I am unable to discuss these arguments here (I will touch on some of the arguments later on in this paper), and have chosen what I believe to be the most important of Hauser's arguments. Hauser presents moral nativism as analogous to the language faculty, therefore I focus on Hauser's claims and arguments that I think provide the most support for his theory.

Dwyer's theory shares many commonalities with Hauser's theory. Rather than reiterating Hauser's arguments discussed above, I will briefly discuss Dwyer's distinctive arguments, especially the poverty of moral stimulus, in favour of a moral faculty with the understanding that her theory encompasses much of Hauser's theory. Dwyer denies that the moral faculty takes the 
form of a module or 'black box' where moral principles reside. In comparing the moral faculty to Chomsky's conception of a language faculty, Dwyer claims that "suffice it to say that one need not think of the language faculty as a module in the Fodorian sense,"15 and she applies the same line of reasoning to the moral faculty. Instead, we can simply think of a faculty as "an abstract posit, ontologically not unlike idealized models elsewhere in science." ${ }^{.16}$ Dwyer claims that a faculty should not be thought of as a black box where principles are inscribed. Rather, the moral faculty should be thought of as an abstract computational system in the brain. Therefore, we should think of the moral faculty in the sense of a Neocartesian faculty, where there need not be a literal or physical module or mechanism in the brain, but an abstract one. ${ }^{17}$

According to Dwyer, we should think of moral nativism as faculty nativism. For instance, in linguistics, faculty nativism entails that "there is an innate faculty of the mind/brain that allows for a certain type of highly constrained cognition. We can talk about the principles that characterize the computation system as being innate." ${ }^{\prime 18}$ E-languages, which are best understood as tangible and historical objects, such as the variety of different languages, ${ }^{19}$ (e.g., English, Spanish, etc.) are not innate, and neither are I-languages, which are the grammars of different languages. E-languages are the different languages that we see throughout the world and that have a long standing history, such as English, Old English, French, and so on. I-languages are the grammars of languages, such as the subject-object-verb arrangement in a language. What is innate are the features (inaccessible principles) that allow for the possibility of E-languages and I-languages. As Dwyer puts it, "the term 'language faculty' has been adopted to refer to these innate features...E-languages, I-languages, linguistic behaviour--none of these can intelligibly be said to be innate. ${ }^{20}$ The same reasoning applies to the moral faculty. Moral norms and moral 
rules are not innate. Rather, the features that allow for moral behaviour and the acquisition of moral norms are innate.

Dwyer claims that "there is good reason to believe that the poverty-of-stimulus considerations arise for moral competence. ${ }^{\prime 21}$ In comparing the moral faculty to Chomsky's language faculty, Dwyer argues that the environment is too impoverished to provide children with enough stimuli to acquire morality and moral norms. If the environment is impoverished, then there must be something innate and domain-specific in the human brain that accounts for moral acquisition. In addition, Dwyer argues that despite the differences, there are many commonalities across cultures in moral decision-making, and these commonalities are a product of the moral faculty; all humans are endowed with a moral faculty, thus there will be many aspects of moral decision-making that are universal. Dwyer argues that the differences across cultures in moral decision-making can be attributed to the social environment, which plays a significant role in shaping the moral faculty, but the differences in moral decision-making are not as drastic as many make them out to be. Similarly, Dwyer claims that because of our moral faculty, there will be many norms that cannot be generated, such as it is good to torture small babies for fun, ${ }^{22}$ in the same way that our language faculty excludes possible languages that have not yet been discovered or created, for example, artificial languages, as learnable.

Dwyer claims that moral decision-making is a product of an evaluation that takes place well below the level of consciousness. As a result, we may appeal to explicit norms when we attempt to justify our answers or even when we make moral judgments, but it would be a mistake to think that the cognitions that are involved in moral decision-making involve reasoning over explicit norms. ${ }^{23}$ This is why we experience moral dumbfounding, a phenomenon that occurs when we cannot seem to justify our moral judgments. On Dwyer's account, moral decision- 
making is not a conscious process despite the reasons we give when justifying our moral judgments.

Jonathan Haidt's conception of moral nativism differs from Hauser and Dwyer's, although there are commonalities with their theories. First, Haidt is arguing in favour of a moral sense theory. Moral sense theorists argue that emotions play an important role in moral development, where moral judgments are grounded in sentiments. Moral judgments, then, are made in the same manner as aesthetic judgments, where specific emotions guide judgments. As Haidt puts it, "judgments about morality have the same status as judgments about humour, beauty, and good writing." ${ }^{24}$ Haidt follows in the footsteps of Hume, arguing that moral beliefs and motivations come from sentiments which give us immediate feelings of rightness or wrongness, and these sentiments are a part of human nature ${ }^{25}$ quick intuitions, moral emotions, and gut feelings guide moral judgments. Haidt argues that the mind is always evaluating and always judging events as good or bad, and the SIM is "founded on the idea that moral judgment is a ubiquitous product of the ever-evaluating mind."26 For example, when "we see an act of violence, or hear about an act of gratitude, we experience an instant flash of evaluation. ${ }^{.27} \mathrm{On}$ Haidt's model, affective responses guide moral judgments; this is where Haidt diverges from Hauser and Dwyer. On Hauser and Dwyer's account of moral decision-making, unconscious reasoning, where emotions are not given a significant role, cause moral judgments. Where Hauser argues that we are not Humean creatures but Rawlsian, where emotions are caused by moral judgments and may sometimes act as constituent components of a moral judgment, Haidt argues that moral judgments are guided by emotions, which play a significant role in moral development. 
Although Haidt grounds his theory in intuitions and emotions, there are commonalities across the SIM and Hauser and Dwyer's account of moral decision-making and moral acquisition. Haidt also believes that the environment is impoverished and cannot provide children with moral behaviour, and agrees that there is a high level of commonality in moral decision-making across cultures. However, rather than attributing the credit to a moral faculty, Haidt claims that we have five domains of moral decision-making, and these five domains are innate foundations in the human brain. The five domains that guide moral decision-making are harm, reciprocity (fairness), authority, community, and purity, and each culture or social group uses all of these domains although different cultures will emphasize some domains more than others. For instance, Haidt claims that conservatives and liberals emphasize some domains more than others, and endorse and promote norms that adhere with those domains. Haidt argues that liberals are more concerned with the principles of harm and reciprocity, while conservatives are generally concerned with all five domains ${ }^{28}$ Individuals and cultures vary in moral decisionmaking because the social environment plays a significant role in shaping, or to use Haidt's term, tuning up, the innate foundations of morality. Education, assistance from peers and adults, the media, etc., play a role in tuning up moral foundations. This is why most individuals that reside in the same society or share cultural values make similar moral judgments, in addition to judging art and beauty in similar manners. In terms of individual differences, Haidt claims that some individuals are born with brains that are prone to experiencing and eliciting stronger intuitions from moral modules, which is why we may witness variation in moral decision-making. ${ }^{29} \mathrm{On}$ Haidt's theory of moral acquisition and moral decision-making, variation in moral judgments is expected. 
Moral judgments are simply gut reactions or intuitions on the SIM; we do not invoke conscious reasoning when we make a moral judgment because we are guided by our emotions and intuitions. Haidt argues that moral intuitions as well as moral emotions occur prior to making a moral judgment, and causes moral evaluation to occur. On Haidt's model, we apply reasoning after the judgment has been made. This is another feature that Haidt's model shares with Hauser and Dwyer's theories. Because we are guided by emotions, we will be unaware of what causes moral judgments, and we will therefore begin to consciously search for answers that support our intuitions after the judgment has already been made. As Haidt states, "moral reasoning is an effortful process (as opposed to an automatic process), usually engaged in after a moral judgment is made, in which a person searches for arguments that will support an already made judgment. ${ }^{30}$ Whereas Hauser and Dwyer argue that we reason consciously and search for reasons that justify our moral judgments due to the process of unconscious reasoning, Haidt argues that we engage in the effortful process of conscious reasoning because we are guided by our passions and must justify them with conscious and meaningful reasons. Therefore, we reason persuasively in order to justify our, in a sense, predetermined moral judgments. I say predetermined because on Haidt's account, moral judgments are made quite effortlessly and intuitively, where our emotions, intuitions, sentiments, and passions are instinctive responses. Although differing on what causes swift and automatic moral judgments, Hauser, Dwyer, and Haidt agree that we are morally dumbfounded when making moral judgments.

To elaborate, Hauser, Dwyer, and Haidt conclude that we experience dumbfounding when attempting to justify our moral judgments or decisions, although they reach the conclusion by means of different analyses. Recall that for Hauser and Dwyer, dumbfounding occurs because after witnessing a moral dilemma, the moral faculty engages in unconscious reasoning, applying 
the inaccessible principles that help determine how we judge a particular moral action, thereby invoking a moral judgment. Due to the fact that reasoning occurs unconsciously and because the principles are inaccessible, we are dumbfounded in explaining our judgment. On Haidt's model, an individual feels a quick flash of an emotion or intuition, say a flash of revulsion at the thought of incest, and reaches the conclusion that incest is wrong even if there are no negative consequences that follow the act. Then, "when faced with a social demand for a verbal justification, one becomes a lawyer trying to build a case, rather than a judge searching for the truth." ${ }^{31}$ However, Hauser, Dwyer, and Haidt all reject the role of conscious moral reasoning when making a moral judgment, denying that conscious moral reasoning plays a role in producing a moral judgment. Rather, conscious reasoning is applied after the judgment has been made in order to justify one's judgment.

Although Haidt's SIM advocates that intuitions are the primary factors in making moral decisions, Haidt does not completely dismiss the role of conscious reasoning in moral decisionmaking, claiming that there are exceptions to unconscious moral reasoning. According to Haidt, at times reasoning plays a role in moral judgments, but only when there are conflicting emotions. However, this is something that we are conscious and aware of, therefore it does not play a part in moral dumbfounding. In addition, conscious reasoning plays a role in moral decision-making, "but only when reasoning runs through other people." ${ }^{32}$ Haidt claims that in a social context we may use reason to persuade peers in believing that specific actions are moral or immoral. Again, this is a conscious act, and is therefore not a part of the moral reasoning described above.

Haidt diverges from Hauser and Dwyer when he makes the claim that humans do not have a moral faculty or a unified moral organ. Rather, on Haidt's account, we come prepared to acquire certain kinds of moral knowledge as well as a resistance to acquiring others. So how 
exactly is Haidt's SIM a moral nativist theory? Essentially, Haidt adopts a Sperberian ${ }^{33}$ view of morality. Haidt claims that "each of our five foundations can be thought of either as a module itself or, more likely, as a 'learning module'--a module that generates a multiplicity of specific modules during development within a cultural context. ${ }^{34}$ Recall that the five domains are harm, fairness, authority, ingroup, and purity. Each domain of morality has an evolutionary story, and "it is quite sensible to suppose that most of the foundations of human morality are many millions of years old, but that some aspects of human morality have no precursors in other animals." ${ }^{35}$ Thus, although Haidt disagrees with Hauser and Dwyer that humans are endowed with a moral faculty, he believes that morality is not placed into the child from outside.

Haidt also recognizes the importance of culture and the environment in moral acquisition. The social environment and culture play a significant role in shaping the five foundations of morality, where the environment interacts with the innate modules to create a specific set of moral norms and intuitions. For example, a child who is raised in a Hindu environment may have the moral intuition that eating meat is immoral (an example of the purity or authority principle at work), and a child raised in a strict Catholic environment may have the moral intuition that abortion is immoral (an example of the authority or harm principle at work). In the same way that linguistic development requires guidance from the local culture to externalize and configure itself properly, morality works in much the same way. ${ }^{36}$

What is more, "each of the five foundations matures and gets built upon at a different point in development...(and) when their minds are ready, children will begin showing concerns and emotional reactions to various patterns in their social world. ${ }^{37}$ In the same way that linguistic maturity and sexual development occur in most individuals at the same age, moral maturity occurs in most individuals at the same age, where each domain of morality is displayed 
at a different point in development. For instance, Haidt claims that the purity foundation develops around the age of seven, which is the age that children acquire the cognitive ability of contamination sensitivity. ${ }^{38}$ This is another feature that Haidt's model shares with Hauser and Dwyer's models. On all three accounts, morality is an innate feature that children will eventually display. In order to be displayed, morality requires guidance from peers, culture, parents, and educators, where these factors shape morality and each moral foundation matures at a different point in development. ${ }^{39}$ In addition, the foundations act as constraints, where certain virtues and norms will never be generated. Hauser, Dwyer, and Haidt agree on the process of moral development and which factors assist in moral development, where morality is acquired with the assistance of innate moral principles and the social environment. This feature of Haidt's SIM is an example of the LA, which will be discussed shortly.

The final component of the SIM that is considered nativist but differs from Hauser and Dwyer's account is Haidt's conception of innate moral modules or foundations. Whereas Hauser and Dwyer argue in favour of innate moral principles, where these principles can take the form of the doctrine of double effect or harm prohibitions against intentional battery, say, Haidt claims that morality is grounded in foundations, with no specific content guiding moral judgments. Rather, there is a significant amount of flexibility in how the foundations can be shaped and which norms can be generated from these foundations. The important difference is that Haidt does not argue in favour of specific innate content. Rather, because the mind is innately prepared with the five foundations, moral norms will be similar across cultures despite the fact that the foundations lack specific moral content. While Hauser and Dwyer argue that moral principles are embedded in an innate moral organ or faculty, Haidt argues that innate moral domains guide moral judgments, where these domains lack specific content. 


\section{The Poverty of Moral Stimulus--Part I of the LA}

Let us now focus on the main arguments in favour of moral nativism, turning our attention first to the poverty of stimulus argument, an argument offered by moral nativists to demonstrate the plausibility of the idea of a moral faculty. The poverty of stimulus argument is defined by John Bolender in "The Genealogy of the Moral Modules" in the following way:

if a perceptual response contains more information than does the stimulus which elicited it, then the perception involves information which the organism has already represented. And if the sum total of the organism's stimuli lacks the information contained in the response, then, according to this argument, the information must be innately represented. ${ }^{40}$

If an individual represents information that cannot be elicited through environmental factors alone, then there must be some kind of innately represented knowledge which allows the individual to represent the information. For example, when children begin to speak, they exhibit and exercise rules of grammar, which given all the rules there are and the very small amount of social interaction children have had by the time they begin speaking make it is highly unlikely that grammar is not innately represented. For example, Hauser et al. note that children invoke the rule of not using the contracted form of "is" whenever they please. Children understand that "although we can say 'Frank is more foolish than Joe is,' we can't say that 'Frank is more foolish than Joe's'. No one taught us this rule. No one listed the exceptions. Nonetheless, young children never use the contracted form in an inappropriate place."41 Therefore, "what the stimulus lacks is produced by the organism from its inner resources. ${ }^{, 42}$ If children exhibit rules of language before the social environment can teach them these rules, that is a ground for believing that these rules are innate. 
In "The Moral Instinct", psychologist and cognitive scientist Steven Pinker points out that four year old children appear to have a conception of moral principles, and are also able to distinguish between conventional and moral norms. Pinker notes that four-year-olds believe it is not okay to hit another child, as well as believing it is not okay to wear pyjamas to school. When children are asked if an authority figure says it is okay to wear pyjamas to school and hit another child if both actions would then be permissible, children respond that it would be okay to wear their pyjamas to school, but still maintain that it is not okay to hit the child, thereby showing the ability to invoke moral principles at a young age, and the ability to distinguish between moral and conventional norms. ${ }^{43}$ Nativists argue that because children are not taught how to distinguish moral norms from conventional norms and do not receive enough stimuli from the environment to make this distinction at the age of four, the ability to make the moral/conventional distinction is innate. Similarly, John Mikhail has pointed out that children display an adequate level of understanding well-developed legal codes, are able to distinguish moral violations from social conventions, and can use intent to distinguish acts with the same results. For example, Mikhail claims that when children are given the scenarios of a man who shoots and kills his victim on the mistaken belief that the victim is a tree stump and a man who shoots and kills his victim on the mistaken belief that killing is permissible, five and six-year-olds distinguish these cases "in conformity with the distinction between mistake of law and mistake of fact." ${ }^{.44}$ Moreover, he notes that in dilemmas such as the Trolley Problem and related dilemmas that distinguish between intended and foreseen consequences, children exhibit the principle of double effect at the young age of eight, while six-year-olds calibrate the level of punishment assigned to harmful facts based on intent, public duty, procedural fairness, and provocation..$^{45}$ According to the moral nativist, it is not possible for children to learn these rules and norms by the time they begin 
displaying moral behaviour. By observing young children and their behaviour, reactions, and judgments when they encounter a moral dilemma, moral nativists claim that it becomes clear that children are making moral judgments that the social environment cannot account for on its own. Thus, there must be some innately represented knowledge.

Linguist Noam Chomsky, father of universal grammar and Principles and Parameters theory, also has a similar conception of morality which stems from his analysis of language. Chomsky argues that like language, there is an innate moral faculty that guides our actions, feelings, intuitions, and judgments in moral decision-making. He asserts that

it cannot be a matter of convention that we find some things right, others wrong. Growing up in a particular society, a child acquires standards and principles of moral judgment. These are acquired on the basis of limited evidence, but have broad and often precise applicability...the acquisition of a specific moral and ethical system, wide ranging and often precise in its consequences, cannot simply be the result of 'shaping' and 'control' by the social environment. As in the case of language, the environment is far too impoverished and indeterminate to provide this system to the child, in its full richness and applicability. ${ }^{46}$

While society teaches us certain rules and norms of morality, it cannot be the foundation of moral decision-making because the social environment cannot supply children with sufficient stimuli to acquire moral behaviour. On this account, the environment works with innate principles in order to produce moral behaviour. Conventional norms shape our innate principles that allow us to make moral judgments, and it is inconceivable that our social environment provides us with these principles on its own. Moreover, the child does not encounter enough stimuli from which they are able to learn moral rules or display moral behaviour. The social 
environment interacts with innate moral principles in order to shape these principles, and it is important to note that the nativist is not disregarding the need for the social environment.

Without the social environment, it would not be possible to shape innate moral principles, where certain parameters are switched on depending on the environment, just as it would be impossible to turn on the switches of the grammar rules associated with Mandarin without a specific social environment. As Chomsky puts it, "language learning is not really something that the child does; it is something that happens to the child placed in an appropriate environment, much as the child's body grows and matures in a predetermined way when provided with appropriate nutrition and environmental stimuli. ${ }^{, 47}$ Similarly, the moral nativist claims that moral learning is not something that the child does, but is something that a child acquires when placed in an appropriate environment. Morality is a feature that humans are innately endowed with, where moral acquisition requires interaction with the environment and cultural divergence is expected.

The objection could be raised that because we all speak different languages, language is solely a product of our social environment. While it is evident that individuals speak different languages, nativists argue that this objection does not show that there are absolutely no innate aspects of language. As Mikhail points out, "what is innate must be consistent with the observed diversity of human languages" diversity across human languages, at the very general level we all have an innate universal grammar that allows us to quickly and effortlessly parse grammar and syntax and comprehend semantics. On the nativist story, claiming that diversity in language entails that we do not have a universal grammar is not a knockdown argument against a language faculty due to the fact that nativists are not neglecting the role of culture, but emphasizing the importance of culture on 
innate principles. For nativists, "the environment determines the way the parameters of universal grammar are set, yielding different languages."

The same line of reasoning applies to morality; what is innate must be consistent with the observed diversity of moral decision-making across cultures. For example, while we all have the same innate moral principles that guide moral decision-making, the social environment will shape these principles to determine which actions are moral and immoral, although at the very general level, principles such as murder, adultery, and promise-breaking are wrong are universal, as Hauser has pointed out. ${ }^{50}$ When judgment boils down to a narrower context, diversity becomes widespread. For example, harm prohibitions differ depending on how societies treat honour. In some cultures, harm is permitted in order to defend one's honour or in order to defend the family's honour. Another example is incest norms, where incest norms differ depending on which family members incest extends to. In some cultures, incest includes first and second cousins, while in other cultures incest includes immediate family members only and marriage between first cousins is permitted or encouraged. For the nativist, harm and incest norms are universal, and these norms are consistent with the observed diversity in human morality.

One worry for the moral nativist is that diversity becomes too widespread when judgment is viewed in a narrower context. With language, diversity is narrow and languages share very specific features, features that are common to all languages studied. The language faculty is embedded with principles that allow for linguistic competence. Linguistic competence allows language speakers to construct sentences, pronounce sentences, and understand sentences (syntax, phonology, and semantics). The rules and principles guiding linguistic competence are universal. This allows humans the potential to learn any language (e.g., French, Hindi, Urdu, English, Italian, Spanish, Portuguese, etc.). Children acquire the native language of their 
community rather than acquiring another language that is not commonly used in their community. This occurs because parameters act like a switch, flipping on the 'English switches' (rules of grammar that pertain to English), let's say, if a child is raised in an English-speaking community rather than flipping on the 'Portuguese switches' (although these languages may share features and can therefore flip on some of the same switches). As Chomsky puts it, "given these properties of language, what we should expect to discover is a system of universal grammar with highly restrictive principles that narrowly constrain the category of attainable grammars, but with parameters that remain open to be fixed by experience. ${ }^{.51}$ If this is the case, there must be universality in language. For instance, there are many restrictions on how words can be moved around in a sentence in order for the sentence to be considered grammatical. These restrictions do not pertain solely to English, but to all languages. ${ }^{52}$ In addition, all languages have principles of locality, where this principle governs the movement of a rule, and all languages also have a principle of opacity, where "variable-like elements can't be free in certain opaque

domains. ${ }^{~} 53$ We do not witness significant differences in languages due to the fact that the factors that govern the system of rules, representations, and principles belong to universal grammar. ${ }^{54}$ According to Chomsky, these rules "are somehow represented in the genotype, along with the instructions that determine that we will grow arms instead of wings, or have a mammalian eye instead of an insect eye.".55

\section{Moral Dumbfounding}

In discussing the poverty of moral stimulus, we have looked at quite a few arguments from language. Let us now turn our attention to moral dumbfounding and the second part of the LA, the principles and parameters theory, and look at how moral nativists use the LA to support moral nativism. The LA, as Dwyer has pointed out, is inspired by the account of dumbfounding 
about language. Learning to speak as quickly as we do, and revealing linguistic rules that we are unable to explain, leaves us dumbfounded in explaining what capacities we have at a very young age that allow for language acquisition. Children construct grammatical sentences without ever learning the rules of grammar. Thus, as Chomsky pointed out, humans must have an innate capacity to parse grammar and syntax and create and comprehend grammatical sentences due to the fact that we express these rules before the social environment can teach us these rules. The same line of reasoning applies to morality. When making moral judgments, we are dumbfounded in explaining what principles we have that allow us to make moral judgments. For instance, when we make the judgment that it is wrong to push the large man off of the footbridge, we are unaware of the moral principle, the doctrine of double effect, that led to the judgment.

As Haidt's SIM has emphasized, we are morally dumbfounded when we make moral decisions; we quickly make a judgment, and then cannot explain how we reached that judgment. For example, Haidt points out that when individuals make a moral judgment such as it is morally wrong to clean a toilet with a nation's flag or that incest is wrong although there are no consequences following the action, individuals simply cannot explain how or why they reached their judgment, but still maintain that the action is wrong. Thus, individuals are morally dumbfounded as to how they reached their judgment, and Hauser, as we have previously seen in the dilemma with UNICEF and the injured child, has attempted to prove dumbfounding as a real phenomenon, where our moral faculty is responsible for dumbfounding. Hauser and Dwyer argue that we are probably dumbfounded for the same reason we are dumbfounded in language; we have innate moral principles that guide moral decision-making, and these principles are processed in a swift and automatic manner, thus when we make an automatic judgment, we do 
not invoke conscious reasoning until after the judgment has been made, hence the dumbfounding.

A worry for the nativist is that we are dumbfounded about many things, not just moral judgments. For instance, we find ourselves dumbfounded in many situations where we intrude in a conversation or blurt out a response to a non-moral dilemma having no idea why we acted in such a manner. Or take the common situation where you ask a teenager why they have a crush on someone and they usually begin to blush and cannot come up with an adequate response. We will explore the possibility that dumbfounding may not be an argument for a moral faculty in Chapter II.

\section{Principles and Parameters-Part II of the Linguistic Analogy}

Let us take a look at Chomsky's conception of Principles and Parameters (PP) with regard to language, and then examine how PP is used in moral nativist theories. It is important to note that when comparing the moral faculty to the language faculty, moral nativists are primarily focusing on PP, although they argue that the structure of universal moral grammar, such as the inaccessible and unconscious principles that guide moral decision-making, are manifested in the same manner as the principles of universal grammar. PP argues that the language faculty is comprised of principles, namely the inaccessible and unconscious principles that guide language acquisition, proper use of grammar, and language comprehension. These principles, "such as the principle for forming causative and other embedded constructions ${ }^{956}$ or the principle that a pronoun must be free in its domain, make up the language faculty and it is what makes language acquisition possible. The parameters can be viewed as switches. The language faculty can be thought of as a complex network with many principles that guide language acquisition, and can be thought of as having a switch box consisting of many switches. The switches must be set in 
one way or another, or the language faculty would not function. According to Chomsky, when the switches "are set in one of the permissible ways, then the system functions in accordance with its nature, but differently, depending on how the switches are set. The fixed network is the system of principles of universal grammar; the switches are the parameters. ${ }^{.57}$ For example, while the principles are innate, unconscious, inaccessible, and guide language acquisition, the parameters act as switches that are fixed by experience. The environment that a child is exposed to, namely the data that is required as input that allows the language faculty to function, set the switches one way or another, where each permissible variety of switch settings determine a particular language. ${ }^{58}$ Similarly, "languages that are historically unrelated may be quite similar if they happen to have the same parameter settings." ${ }^{.59}$ Thus, certain parameters are 'switched on' depending on the child's environment. If a child is raised in an English-speaking environment, then the parameter 'a head must precede its complements' is switched on in order to provide the child with the proper rules of grammar used in their environment. The environment works as the stimuli determining which switches of universal grammar will be operative in a child. A child raised in a Japanese-speaking environment will have turned on different switches than a child raised in an English-speaking environment. It is also important to note that nativists with regard to both language and morality argue that without the social environment, acquiring language or moral norms would not be possible, and nativists recognize the importance of the environment in shaping innate language principles and innate moral principles.

With regard to the moral faculty, PP works in much the same manner. The innate, inaccessible, and unconscious principles that guide moral decision making are responsible for moral acquisition. The parameters, switched on by the environment, determine which set of moral norms children come to accept. For example, a child raised in Japan will have a different, 
although possibly similar, set of moral norms than a child raised in Boston, and a child raised in 1950 Louisiana may have a different set of moral norms than a child raised in 2011 Louisiana. However, these norms share common features despite their differences. As Hauser puts it, "the moral faculty constrains the range of both possible and stable ethical systems, ${ }^{, 60}$ in the same way PP with regard to language constrains the number of possible grammars. The language faculty consists of possible grammars that a child can acquire, providing options within a restricted range, where acquiring an artificial language would be difficult because it is not a product of the language faculty. This is why Dwyer argues that certain moral norms can never be generated; simply, our moral faculty provides options for morality in a rather restricted range, where the moral faculty constrains the number of possible moralities.

In line with the LA and PP, Hauser argues that in the same way grammaticality judgments are delivered rapidly, spontaneously, and with little reflection, ethical judgments are delivered in the same manner. Emphasizing the similarities between language and morality and capturing the essence of using PP, Hauser claims that

in the same way that grammaticality judgments emerge from a universal moral grammar of principles and parameters, the Rawlsian creature's ethicality judgments would emerge from a universal moral grammar, replete with shared principles and culturally switchable parameters. From this perspective, each culture expresses a specific moral grammar. The Rawlsian creature therefore places constraints on the range of possible variation, including the range of potential moral systems. ${ }^{61}$

This quote captures the reasoning behind using PP perfectly and emphasizes the supposed relation between language and morality. 
Hauser and colleagues point out that there is also a distinction between expressed and operative knowledge which captures the essence of PP. Expressed knowledge can be viewed as the product or the rules of operative knowledge. Operative knowledge is knowledge that is automatic and unconscious; it is information that we are equipped with that causes expressed knowledge. Hauser et al. provide an example of expressed and operative knowledge in their article "Reviewing Rawls's Linguistic Analogy". Here, Hauser et al. give the example of a baseball game, where expressed knowledge would include such things as our knowledge that a fly ball travels a parabolic arc describable by a quadratic mathematical expression...(while) operative knowledge includes such things as our knowledge of how to run to just the right spot on a baseball field in order to catch a fly ball...our brain must be carrying out these computations in order for us to get the right spot, even though, by definition, we can't articulate the principles underlying this knowledge. ${ }^{62}$

Expressed knowledge can be seen as a rule, or product, that is produced from operative knowledge, where the social environment helps shape operative knowledge. In both morality and language, the innate principles guiding moral judgments and grammar are operative knowledge, where moral norms and the language one speaks are expressed knowledge. Operative knowledge is also the knowledge that is universal across individuals, whereas overt behaviours stemming from operative knowledge can differ.

Briefly, we need to distinguish moral principles from moral rules for clarification purposes and to give moral nativism a fair chance in showing its plausibility. With regard to our language faculty, the principles are conceived in the form of an innate universal grammar, where the rules of grammar and syntax are the innate principles which we cannot consciously access 
and which we are not consciously aware of. For example, we understand that the expression colourless green ideas sleep furiously is a grammatical sentence, yet it makes no sense. While we are able to parse the syntax of the sentence, we cannot infer what is meant by these words. Chomsky came up with this sentence to "not only show that nonsense can be grammatical but also to show that improbable word sequences can be grammatical." ${ }^{63}$ In addition, when English speakers hear the sentence Yoko Ono will talk about her husband John Lennon who was killed in an interview with Barbara Walkers, ${ }^{64}$ although the meaning is ambiguous because of the way the words are joined together syntactically, we are still able to grasp the content, namely that Yoko Ono will speak to Barbara Walkers about the death of her husband who was killed, rather than Yoko Ono speaking about her husband who was killed by Barbara Walkers while being interviewed by her. Our language faculty provides us with the ability to parse the sentence syntactically in order to determine the verb phrase, noun phrase, prepositional phrase, etc., and we also infer what exactly the speaker is trying to tell us from uttering this sentence, despite the ambiguity of it. ${ }^{65}$ Moreover, as Steven Pinker notes, "the...consequence of the design of grammar is that it is a code that is autonomous from cognition. A grammar specifies how words may combine to express meanings; that specification is independent of the particular meanings we typically convey or expect others to convey to us."

Moral nativists argue that we can view moral principles and moral rules in the same manner. For example, while the innate principle of harm can be conceived as 'do not unnecessarily harm innocent individuals,' the principle is shaped into a moral rule depending on one's social environment. For example, culture A may invoke the harm principle only to the extent that they do not unnecessarily harm individuals unless one must be sacrificed for religious purposes. Culture B may invoke the harm principle to the extent that individuals must not be 
harmed unless in self-defense. So while the moral principle harm is innate, the rule, namely what society shapes of that principle (which parameters are switched on), is not innate but is a product of the innate principle. Applying the LA, where the language Mandarin (an E-language) is not innate, neither is the moral rule "Thou shall not harm", but the principles guiding Mandarin and the principle guiding the rule "Thou shall not harm" are innate.

It is important to point out that "no advocate of the LA claims that morality is, in every respect, similar to language, a contention that is clearly incoherent, given their transparent functional differences. ${ }^{167}$ However, moral nativists argue that there are striking similarities between innate language principles and innate moral principles. In terms of the differences, we can claim that there is a right and wrong way, according to a specific language, to grammatically structure a sentence, as well as claiming that there is a right or wrong way to turn present tense verbs into past tense verbs. There are specific rules that are followed in language construction, thanks to our language faculty. With a moral faculty, right and wrong is not exactly a clear notion. While we have innate moral principles that guide moral decision-making, this does not entail that the principle of harm or community will tell us that action $\mathrm{A}$ in situation $\mathrm{X}$ is right, while a similar action B is wrong in situation Y. With moral principles, because they are inevitably shaped by culture and the environment more so than language and are therefore open to interpretation, it is difficult to unambiguously assert whether an action is morally right or wrong, although with language, we can decisively assert that a sentence is grammatical or ungrammatical. And of course, with language, depending on the society and which language is spoken, the arrangement of the subject-object-verb order will differ, however, the innate principles of grammar will always remain the same. With morality, the innate principles are universal, but what is deemed as morally right or wrong depends on which moral principle is 
triggered and how the social environment shapes moral principles. However, moral nativists maintain that the LA is useful when arguing in favour of innate moral principles due to the fact that in both language comprehension and moral decision-making, we are dumbfounded as to how certain conclusions are reached, the innate structures are manifested in much the same manner, both are a universal feature of being human, and while the principles are innate, culture plays a significant role in shaping the principles.

\section{Universality and Innateness}

Now that we have fleshed out what moral nativism consists of and have examined the three main components of moral nativism, let us take a look at a final argument that moral nativists put forth in support of a moral faculty (or in Haidt's case, five domains of morality that take the form of Sperberian modules). We have already looked at some examples in favour of universality in moral decision-making, such as the Trolley Problem. But moral nativists do not only look to hypothetical thought experiments to determine whether there is universality in moral decision-making. Rather, they look for universality in moral decision-making across cultures that appear to have different sets of moral norms.

As previously mentioned, moral nativists do not reject the role of the environment in shaping moral norms. Rather, they argue that the environment plays a significant role in moral acquisition, where the environment shapes moral principles and determines which set of moral norms a child comes to acquire. According to the moral nativist, the fact that children in North America and Eastern Asia will acquire different sets of moral norms does not weaken the nativist position. Rather, despite these differences, there is universality in moral decision-making.

In an attempt to prove that there is universality in moral decision-making despite crosscultural differences, Hauser argues that killing, causing pain, lying, promise breaking, cheating, 
and adultery are all universally forbidden ${ }^{68}$ In addition, he claims that like all other rules, norms, and conventions, these moral prohibitions have exceptions, so that although killing may generally be forbidden in all cultures, most cultures recognize that there are some conditions where killing may be permitted or may be obligatory. For example, he claims that in instances of war, intolerable pain, or self-defense, killing may be justifiable, or in some cultures if a wife is caught engaging in adulterous behaviour or is raped, the wife's relatives have a moral obligation to kill her in order to erase the family's shame. ${ }^{69}$

Hauser claims that what allows for both universality and cultural variation are the unconscious principles guiding moral decision-making. What is more, in searching for these principles Hauser concludes that "if, as Rawls intuited, the analogy between morality and language holds, then our common sense accounts will be insufficient." ${ }^{70}$ Therefore, it is the interaction of these undiscoverable principles and the environment that allows for variation in moral decision-making, where variation is attributed to the social environment and does not undermine the plausibility of a moral faculty. Rather, the moral principle of harm is still universal across cultures, but the way in which each culture shapes the harm principle will allow for variation in harm norms. Because innate moral principles must interact with the environment in order to function, the social environment shapes the innate principles and produces moral norms and rules. While all individuals have the same innate moral principles, they will not come to favour or accept similar moral norms; the environment interacts with innate moral principles, shaping moral principles to produce various moral norms, which explains why harm norms differ across cultures. In the same way that innate language principles need the environment to trigger specific switches that give rise to a specific language, innate moral principles need the environment to trigger specific switches that give rise to specific moral norms. While languages 
and moral norms are not innate, the innate principles are. For the moral nativist, differences in moral decision-making are a product of the environment, and there will be universality in moral decision-making because all moral norms stem from innate moral principles.

Let us briefly return to the example of feeling morally obligated to help the injured child we encounter but not feeling morally obligated in donating fifty dollars to UNICEF, where Hauser uses this dilemma as an example of universality in moral decision-making. Hauser argues that because of the universal way in which individuals judge this moral dilemma, there must be a corresponding innate principle in our moral faculty, such as "if we can directly prevent, with a high degree of certainty, something bad without sacrificing anything of comparable significance, we are obliged to do it." ${ }^{, 71}$ However, the social environment sets the parameters on the norms that emerge from the innate principle, which is why we see variation in moral decision-making across cultures, while at the same time witnessing universality in moral decision-making in specific moral dilemmas.

With regard to the Trolley Problem and Hauser's web-based experiment that suggests individuals respond in the same manner to this dilemma irrespective of location, Hauser claims that we respond in the same manner because of our innate principle of harm, where we can view the principle of harm, at least in the context of the Trolley Problem, as the principle of double effect. The principle of double effect is a moral principle that prohibits acts of harm if the consequences of the action are intentional, but does not prohibit harm if the consequences of the action are not intentional and if the actions foreseeable effects outweigh the foreseeable bad effects. ${ }^{72}$ In addition, although we may respond in a similar manner in hypothetical moral dilemmas such as the Trolley Problem, there is variation across cultures with regard to the harm principle, and Hauser argues that in line with the LA, "we expect differences between cultures 
with respect to how they set the parameters associated with principles for harming and helping others." ${ }^{.73}$ Similarly, nativists argue that all cultures have sharing norms which stem from an innate principle in the moral faculty. While all cultures have norms pertaining to sharing, they will differ on what they consider fair. Thus, while at a general level the principles of harm and sharing are universal, we will see variation across cultures, and for moral nativists this does not weaken the nativist position, but simply reinforces the role of culture and the environment in shaping moral principles.

Haidt claims that "despite the obvious cultural variability of norms and practices, there is a small set of moral intuitions that is easily found in all societies. ${ }^{, 74}$ As previously mentioned earlier on in this chapter, this small set of moral intuitions include harm, fairness, authority, purity, and community. Although Haidt notes that some social groups, such as liberals, dismiss the last two intuitions as matters of social conventions, most cultures and social groups see matters of community and purity as parts of their moral domain, thus they are included in the small set of moral intuitions that are found in all societies. ${ }^{75}$ For example, Haidt notes that the community and purity foundations are norms that are widespread but are not mentioned in many studies concerning morality. However, Haidt argues that authority and purity norms are widespread and are common in the world's cultures, and should therefore be considered innate domains. Thus, "these five sets of intuitions should be seen as the foundation of intuitive ethics, ${ }^{, 76}$ where these intuitions guide moral decision making in all cultures and social groups. On Haidt's model, universality is a product of the five sets of intuitions, where the first three intuitions are shaped by evolution and are shared with primates, and the last two are recent developments in the moral domain and are specific to human morality. In addition, the fact that these intuitions or foundations of morality are thought of as modules in the mind that develop 
within a specific context is what makes moral decision-making universal. Because all humans are endowed with innate moral modules, cultures will have universal norms where the content of norms differs depending on the context and environment.

One example Haidt gives in favour of universality across cultures is the game of cooties. He argues that all over the United States, children play the game of cooties, where cooties "are a kind of invisible social germ," receiving a 'cooties shot'. Haidt claim that although the cooties game is not supported by the media and is opposed by adults, the game persists for many decades and centuries, while some games do not get transmitted at all. In addition, Haidt claims that the game of cooties is found in many cultures that are not closely related, and is a game that is "persistent, stable, and ubiquitous." ${ }^{, 78}$ What is more, Haidt claims that the cooties game "is a product of the maturation and elaboration of the purity foundation. When children acquire the cognitive ability of contamination sensitivity around the age of 7 , they begin applying it to their social world." Thus, Haidt concludes that because of the purity domain, the game of cooties is present; if humans did not have a purity foundation that guides moral decision-making, the cooties game would not exist nor would it get transmitted over decades. ${ }^{80}$

In line with Hauser and Dwyer's argument, Haidt argues that there are certain virtues that simply are not learnable. Arguing against Patricia Churchland's claim that any virtue a society reinforces will be the virtues or norms that the children in the society develop, ${ }^{81}$ Haidt argues that due to our five foundations of intuitive ethics, there are simply some norms or virtues that will never be developed. Moreover, Haidt claims that "as long as people have intuitions about harm and suffering, anyone who acts to relieve harm and suffering will trigger feelings of approval. ${ }^{\text {82 }}$ Thus, the act of kindness receives moral approval, while acts that inflict suffering 
receive moral disapproval, and Haidt claims that although what it means to be kind may vary across cultures, there will in fact be a resemblance across cultures that determine what constitutes an act of kindness. ${ }^{83}$ The same goes for the other moral intuitions as well. For example, most individuals agree that incest is wrong, even if there are no negative consequences. ${ }^{84}$ However, determining what counts as incest is dependent upon the social environment. Some cultures do not prohibit sexual relations with first cousins, while some cultures believe that sexual relations with a childhood friend counts as a form of incest.

In addition, with regard to what appears to be enormous differences in moral norms across cultures, such as norms of honour, Haidt claims that for complex norms, the norm matches with one of the five foundations of moral decision-making, simply because it would be impossible for a culture to construct a norm that does not mesh with one of the foundations. Because these foundations are moral foundations and are what causes the emergence of moral norms, all moral norms must refer back to one or more of the innate domains of morality. For instance, the norm that a daughter shames her family because she is a victim of rape or the norm that an honourable man avenges insults, meshes with one or more of the five foundations of morality, such as purity or fairness. ${ }^{85}$

In explaining why we witness variation in moral decision-making despite the guidance of the five foundations of intuitive ethics, Haidt claims that we can attribute variation to learning, influence from peers and the media, and practice. Arguing that "some people are simply born with brains that are prone to experience stronger intuitions from individual moral modules, sociocultural factors assist these intuitions in 'tuning up' as children develop the skills that are unique to the intuitions their biology favours. Individual differences can be explained by the fact that some individuals are more prone to experiencing some intuitions stronger than others, where 
the social environment plays a significant role in shaping intuitions. Thus, Haidt concludes that intuitions guide the development of norms and virtues, where cultures will differ in moral decision-making due to the fact that groups of individuals may be prone to favouring some moral intuitions over others.

\section{Conclusion}

In this chapter, I have provided a detailed discussion of moral nativist theories and the main components of moral nativism. In the next chapter, we look at specific problems associated with the poverty of moral stimulus argument, moral dumbfounding, and the LA, as well as looking at why it is implausible to make a jump from universality to innateness.

\section{Notes}

${ }^{1}$ Steven Pinker, "The Moral Instinct," New York Times, January 13, 2008.

${ }^{2}$ Hauser et al., 159.

${ }^{3}$ Marc Hauser, Moral Minds (New York: HarperCollins Publishers, 2006), 111-159.

${ }^{4}$ Hauser, xvii.

${ }^{5}$ Ibid.

${ }^{6}$ Hauser, 38.

${ }^{7}$ Hauser, xviii.

8 Hauser, 7.

9 Jonathan Haidt, "The Emotional Dog and its Rational Tail: A Social Intuitionist Approach to Moral Judgment," Psychological Review 108 (2001): 5.

${ }^{10}$ For the full example and similar examples, see Hauser 1-48.

${ }^{11}$ Hauser, 30.

${ }^{12}$ Hauser, 53.

${ }^{13}$ Hauser, 42.

${ }^{14}$ Hauser, 14.

${ }^{15}$ Susan Dwyer, "How Not to Argue that Morality Isn't Innate: Comments on Prinz" as in article in Walter SinnottArmstrong, Moral Psychology: The Evolution of Morality: Adaptations and Innateness vol. 1 (Cambridge: MIT Press, 2008), 410.

16 Ibid.

${ }^{17}$ Although I cannot discuss the issue here, I briefly note a few questions that are left open with Dwyer's view of a moral faculty not being postulated to be in the form of a module: How are the moral principles triggered? More specifically, what mechanism in the brain triggers the principles? What ensures that other innate principles in the brain are not interfering with our innate moral principles, thus giving us a skewed perception of moral judgments? How can principles alone automatically compute and output a moral judgment? Which mechanism is needed in order to ensure that the moral principles are at work? 
18 Dwyer, 410.

${ }^{19}$ Dwyer, 409.

${ }^{20}$ Dwyer, 410.

${ }^{21}$ Dwyer, 411.

22 Dwyer, 414.

${ }^{23}$ Dwyer, 413.

${ }^{24}$ Jonathan Haidt and Fredrik Bjorklund, "Social Intuitionists Answer Six Questions about Moral Psychology" as an article in Walter Sinnott-Armstrong, Moral Psychology: The Cognitive Science of Morality: Intuition and Diversity vol. 2 (Cambridge: MIT Press, 2008), 214.

${ }^{25}$ Haidt and Bjorklund, 185.

${ }^{26}$ Haidt and Bjorklund, 188.

27 Ibid.

${ }^{28}$ See Jonathan Haidt and Jesse Graham, "When Morality Opposes Justice: Conservatives Have Moral Intuitions That Liberals May Not Recognize," 2006.

29 Haidt and Bjorklund, 210.

${ }^{30}$ Haidt and Bjorklund, 189.

31 Haidt, 2.

32 Haidt, 7.

${ }^{33}$ Sperberian simply refers to the view of anthropologist Dan Sperber.

${ }^{34}$ Haidt and Bjorklund, 205.

${ }^{35}$ Haidt and Bjorklund, 204.

${ }^{36}$ Haidt and Bjorklund, 206.

${ }^{37}$ Ibid, parenthesis mine.

${ }^{38}$ Haidt and Bjorklund, 207.

${ }^{39}$ Haidt and Bjorklund, 206.

${ }^{40}$ John Bolender, "The Genealogy of the Moral Modules," Minds and Machines 13 (2003): 234.

${ }^{41}$ Marc D. Hauser, Liane Young, and Fiery Cushman, "Reviewing Rawls's Linguistic Analogy" as in article in Walter Sinnott-Armstrong, Moral Psychology: The Cognitive Science of Morality: Intuition and Diversity vol. 2 (Cambridge: MIT Press, 2008), 111.

${ }^{42}$ Noam Chomsky, Rules and Representations, (New York: Columbia University Press, 1980), 36.

${ }^{43}$ Steven Pinker, "The Moral Instinct."

44 John Mikhail, "The Poverty of the Moral Stimulus" as in article in Walter Sinnott-Armstrong, Moral Psychology: The Evolution of Morality: Adaptations and Innateness vol. 1 (Cambridge: MIT Press, 2008), 354.

${ }^{45}$ Mikhail, 354-355.

${ }^{46}$ Noam Chomsky, Language and Problems of Knowledge: The Managua Lectures (Cambridge: MIT Press, 1988), 152-153.

${ }^{47}$ Chomsky, 134.

${ }^{48}$ Mikhail, 355.

${ }^{49}$ Chomsky, 134.

${ }^{50}$ Hauser, 48.

${ }^{51}$ Chomsky, Rules and Representations, 66.

${ }^{52}$ Andrew Brook and Robert J. Stainton, Knowledge and Mind : A Philosophical Introduction (Cambridge: MIT Press, $2000), 47$.

${ }^{53}$ Chomsky, 91.

54 Ibid.

55 Ibid.

${ }^{56}$ Chomsky, 14

${ }^{57}$ Chomsky, 62-63.

${ }^{58}$ Chomsky, 63.

59 Ibid.

${ }^{60}$ Hauser, 54. 
${ }^{61}$ Hauser, 43, 44.

62 Hauser et al., 109, parentheses mine.

${ }^{63}$ Steven Pinker, The Language Instinct: How the Mind Creates Language (New York: HarperCollins, 1994$), 85$.

${ }^{64}$ I borrow this example from Pinker, 1994.

${ }^{65}$ It is also important to point out that while our language faculty is responsible for parsing syntax and disambiguating multiple meanings of one sentence, we may have a comprehension/metacommunicative mechanism or submodule that assists with this task. See Sperber and Wilson 2002 for a detailed analysis.

${ }^{66}$ Pinker, 78.

${ }^{67}$ Susan Dwyer and Marc Hauser, "Dupoux and Jacob's Moral Instincts: Throwing Out the Baby, Bathwater and the Bathtub" Trends in Cognitive Science 12, no.1 (2007): 2.

${ }^{68}$ Hauser, 48.

${ }^{69}$ Ibid.

${ }^{70}$ Ibid.

${ }^{71}$ Hauser, 65.

72 Hauser, 124.

${ }^{73}$ Hauser, 129.

${ }^{74}$ Haidt and Bjorklund, 202.

${ }^{75}$ Haidt and Bjorklund, 203.

${ }^{76}$ Ibid.

${ }^{77}$ Haidt and Bjorklund, 206.

${ }^{78}$ Haidt and Bjorklund, 207.

${ }^{79}$ Ibid.

${ }^{80}$ Ibid.

${ }^{81}$ Patricia Churchland, Braintrust (New Jersey: Princeton University Press, 2011), 166-184.

${ }^{82}$ Haidt and Bjorklund, 208.

83 Ibid.

${ }^{84}$ Haidt, 2.

${ }^{85}$ Haidt and Bjorklund, 208.

${ }^{86}$ Haidt and Bjorklund, 210. 


\section{Chapter II: Introduction}

In Chapter I we looked at moral nativism and discussed three main components of the argument for moral nativism, the poverty of moral stimulus argument, moral dumbfounding, and

PP. We also looked at arguments in favour of universality, where nativists argue that universality in moral decision-making is a product of a moral faculty or innate moral foundations. This chapter critically examines moral nativism. More specifically, I examine the nativist theories put forth in Chapter I and determine the plausibility of moral nativism. I argue that the poverty of moral stimulus argument, moral dumbfounding, the PP, and the argument from universality are problematic in supporting moral nativism. In addition, I analyze Haidt's SIM and argue that the five innate foundations of morality do not plausibly account for moral acquisition. Moreover, in analyzing Haidt's SIM I emphasize the nonnativist components of his model and discuss the similarities between the SIM and the nonnativist theories that will be discussed in Chapter III. Rather than analyzing and determining the plausibility of each nativist theory discussed in Chapter I, I analyze the components of moral nativism. The three theories argue for moral nativism in similar manners, putting forth arguments in favour of the ideas of the poverty of moral stimulus, moral dumbfounding, PP, and universality.

\section{Problems with the Poverty of Moral Stimulus Argument}

Let us begin by exploring the possibility that our stimuli may not be impoverished in acquiring moral behaviour and determine whether morality can be acquired solely through environmental factors.

Recall from the Introduction that moral nativists are concerned with defending content nativism and nonnativists do not take issue with capacity nativism, namely that humans are predisposed to have certain capacities, such as a ToM, which is a theory of mind mechanism (or 
simulation), that contributes to moral decision-making, or that humans have a capacity to moralize. That being said, we must first examine whether content nativism is correct. First, we will determine whether the argument from the poverty of moral stimulus is plausible. Chomsky, among other theorists, argues that children begin speaking at a very tender age and given the small amount of exposure to the environment children have had by the time they begin speaking, there must be some sort of innate content that allows for language acquisition. With regard to language acquisition, our stimuli are impoverished, as we cannot account for language acquisition or explain language acquisition by appealing to the environment. For example, children express rules of grammar that they have never heard adults use and begin speaking in full sentences before they have had exposure to a significant number of words and the rules of sentence structure. Therefore, the environment cannot be appealed to when explaining how children acquire language. Rather, the only plausible explanation is that a large part of language is innately represented in the mind.

Can the same line of reasoning be applied to morality? Recall John Mikhail's argument that moral judgments cannot be made by appealing to innate biases, simple imperatives, or the environment. He argues that moral intuitions are complex and that the "moral competence of both adults and children exhibits many characteristics of a well-developed legal code, including abstract theories of crime, tort, contract and agency." Because the emergence of such knowledge cannot be explained by appealing to the environment, associative learning, ${ }^{2}$ imitation, or explicit instruction, there must be an innate faculty that accounts for this information, as is the case with language. Mikhail argues that three and four year olds use both intent and purpose to distinguish acts with similar consequences, as is the case with the Trolley Dilemma, calibrate the level of punishment for harmful acts on the basis of provocation, intent, and purpose, and display the 
doctrine of double effect a little later on in childhood, at the age of eight. Thus, the environment must be impoverished with regard to moral acquisition, even though accepting a doctrine where "children possess an elaborate system of natural jurisprudence and an ability to compute mental representations of human acts in legally cognizable terms" ${ }^{\prime 3}$ may be difficult.

One problem with this view is that, as Mikhail points out, it is difficult to accept that children possess an innate elaborate system of the abilities mentioned above. These abilities may be by-products of other capacities and can be learned through explicit instruction or associative learning. In addition, and as we will see in Chapter III, we may have innate biases that account for these abilities.

Let us take a look at whether the ability to use intent and purpose to distinguish acts with similar consequences, or similarly the doctrine of double effect, is an ability that is specific to the moral faculty. Mikhail makes the strong claim that the content of the doctrine of double effect, that an act with a foreseen but not an intended negative consequence is permissible while an act with a foreseen and an intended negative consequence is impermissible, is innate, and the abstract concepts that constitute the doctrine are also innate. However, this doctrine, as well as the ability to use intent and purpose to distinguish acts with similar consequences, may be seen as part of another innate capacity, such as a ToM (or simulation) or a theory of action. Sripada notes that "to the extent that young children master these concepts without much in the way of teaching, then this fact suggests that theory of mind and theory of action are importantly innate. ${ }^{4}$ Recall that nonnativists are not denying that certain capacities that assist in moral decision-making are innate; they are not rejecting capacity nativism. What nonnativists are rejecting is that there is a moral faculty that is innate, where moral decision-making cannot be made without such a faculty. Therefore, the nonnativist concedes that a ToM or a theory of 
action may assist in making moral judgments, but may not be particular to a moral faculty, as these capacities are also used when not making moral judgments. What is more, Hauser notes that certain capacities, such as a capacity that allows children to comprehend the world ${ }^{5}$, a sense

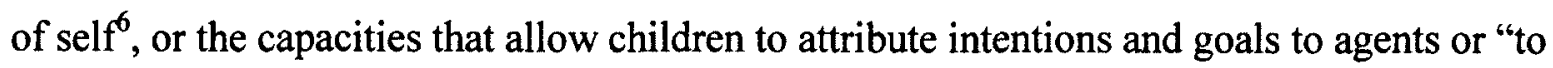
predict patterns of affiliation based on actions associated with positive or negative emotions"7 are not specific to a moral faculty but assist in moral decision-making. Hauser also notes that without these capacities, individuals "would never make it into the moral arena,"8 yet these capacities alone cannot make individuals moral creatures, and Hauser argues that a moral organ with the assistance of these other capacities allow for moral acquisition.

Nativists also argue that our stimuli are impoverished in acquiring morality because children distinguish between moral and conventional rules at a relatively young age. However, parents and educators may enforce moral norms and conventional norms differently, and by associating specific attitudes and behaviours from peers and educators with a specific reaction, children may be able to learn that norms that are received negatively and with negative punishment, are authority independent, and are very serious. They are moral norms rather than conventional.

For instance, Prinz notes that we do not need to posit an innate moral faculty to explain the ability to distinguish between moral and conventional norms because parents and educators react differently in response to transgressing moral norms and conventional norms. With a harm norm such as 'do not hit', parents respond in the following manner(s) when children violate this norm: parents usually exert power and get angry with their child and threaten them with a timeout or no dessert after dinner; there may be love withdrawal where a parent refuses to play with a child if the child is misbehaving; and there is also induction of empathetic distress, where 
parents will explain to a child that they have made another child cry by hitting them. ${ }^{9}$ According to Prinz, all three methods are effective in enabling children to distinguish between moral and conventional norms. Prinz claims that power assertion helps a child recognize that the rule or norm in question is serious, love withdrawal is a form of associative learning where a child will learn that love was withdrawn because they did something bad or wrong, and induction of empathy allows the child to associate the act with distress, and teaches the child that hitting is wrong because it causes sadness or misery in the victim. ${ }^{10}$ Prinz calls these methods internalization, where "an internalized rule is just a rule of conduct toward which one has an emotional attitude that leads one to be motivated to act in accordance with the rule and to negatively regard others who fail to act in accordance with the rule." conventional rules, where parents are likely to get angry when a child transgresses a conventional norm, parents will not get angry to the same extent or react in the same manner when punishing a child for transgressing a conventional norm as they do with punishing a child for transgressing a moral norm. For instance, parents probably will not respond with power assertion, love withdrawal, and will not be able to induce empathetic distress because the child's behaviour is not harmful to the same extent as when violating a moral norm such as 'do not hit others'.

Thus, conventional rules and moral rules are enforced in different manners and are treated differently when transgressed. The differences in how moral norms and conventional norms are enforced can account for the ability to distinguish between moral and conventional norms at a young age. Children receive a significant amount of feedback when transgressing both moral and conventional norms, and this feedback begins at a very young age. The ability to 
make this distinction may not be a product of a moral faculty, but simply a product of our environment and upbringing.

Similarly, some conventional norms, such as that it is wrong to spit into a napkin at a dinner table, may be conceived of as moral norms in some cultures if transgressing the norm is received in a similar fashion to transgressing a moral norm, and if it elicits an affective response. As both Prinz and philosopher Shaun Nichols have pointed out, if transgressing a conventional norm elicits an affective response, it will probably be treated as a moral norm; affective responses play a significant role in treating certain disgust violations as immoral rather than morally neutral. Thus, eating meat or spitting into a napkin at a dinner table may be seen as morally wrong in some cultures if they trigger an affective response. As Nichols points out in Sentimental Rules, if parents and educators treated and enforced certain etiquette norms in the same manner as harm norms, children will come to have a moral attitude toward those etiquette norms, treating them as a moral norm rather than a conventional norm. What is more, a norm will have a greater chance of survival if it is backed by affect, more particularly, negative affect. Societal and biological factors will also have an impact on which norms get transmitted, but affect plays a significant role in transmitting norms and determining the survival of norms. ${ }^{12} \mathrm{We}$ will return to this theory in Chapter III.

Another reason it may be plausible to argue that our stimuli are impoverished with regard to language acquisition but not moral acquisition is that in the process of moral acquisition, the learning target is less complex than in the process of language acquisition. In order to argue in favour of the view that our stimuli are impoverished with regard to moral acquisition, the moral nativist must prove that, like language, there is a gap between the learning target, namely moral acquisition, and the learning resources available. As previously mentioned, it is plausible to 
assert that there is a gap between the learning target and learning resources with regard to language acquisition. However, it may not be plausible to suppose that there is a gap between moral acquisition and the learning resources available to an individual.

Sripada points out that

an important disanalogy between language learning and moral norm learning is that in the case of moral norm learning, the learning target is far simpler than in the case of language. Moral norms are not abstruse and far removed from experience in the same manner as the hierarchical tree structures and recursive rules of human grammars. ${ }^{13}$ Unlike language learning, moral norm learning requires that children acquire an understandable set of imperatives and rules, such as 'do not hit or push other children', where more complex moral norms such as 'adultery is wrong' are learned later on in life after basic moral imperatives are learned and "after the conceptual resources needed to understand such rules are firmly in place." 14 Thus, when children learn the basic set of moral imperatives, such as hitting is bad and cheating is wrong, they are able to learn more complex moral norms later on in life that build on the basic set of moral imperatives learned in childhood. Unlike the case of language acquisition, children are explicitly instructed and told what is permissible and impermissible. Because the learning target is not too complex with regard to moral acquisition, and because, as argued above, there are sufficient stimuli and resources available to a child, the poverty of moral stimulus argument may not be as plausible as the moral nativist believes it to be. Rather, the gap between the learning target and learning resources seems to be nonexistent. Thus we have grounds for rejecting the poverty of moral stimulus argument.

Another philosopher who argues in favour of the poverty of moral stimulus argument is Gilbert Harman. Harman argues that with complex moral principles, such as the doctrine of 
double effect, there aren't sufficient learning resources for acquiring this principle as well as other complex principles. In the same way that some linguistic principles are highly complex and not explicitly known to speakers, the same may be true of moral principles if they are equally complex and not explicitly known or taught to individuals. Harman also argues that "it is unlikely that children require explicit instruction in order to acquire a first morality, any more than they require explicit instruction in order to acquire language, although interaction with others may be necessary in both cases." ${ }^{.15}$ Let us examine both of Harman's arguments in turn.

First, Harman argues that with complex moral principles, such as the doctrine of double effect, there aren't sufficient learning resources for acquiring this principle as well as other complex principles. Harman argues that moral nativism is able to explain how children acquire complex moral principles that appear universal. However, as already explained above, most individuals may be repulsed by the idea of physically harming others, and in situations where individuals are asked to imagine being closer to the victim or physically harming the victim, individuals may be more emotionally charged than they are when thinking about dilemmas where they have no direct contact or are at a distance from the victim.

In the following chapter, we will see that emotions may be constituent components of moral decision-making, and that if a dilemma is emotionally charged, an individual may view the dilemma in question as morally reprehensible. In addition, Sripada notes that the doctrine of double effect and similar capacities are best understood and cognized in terms of a ToM capacity or a theory of action. If children do in fact master these concepts without being exposed to stimuli that allow them to acquire the principle, then a ToM and theory of action are importantly innate, but it does not follow that the doctrine of double effect is an innate moral principle. 
Second, Harman claims that it is unlikely that children require any explicit instruction in acquiring morality. This claim is extremely hard to swallow. It seems very unlikely that children do not require any explicit instruction in order to learn right from wrong. For instance, it seems unlikely that a toddler will innately know that pushing or stealing a toy is wrong. If toddlers did not need explicit instruction to acquire these norms, then toddlers would not push other toddlers or take toys that did not belong to them. Parents are always correcting children on what is morally permissible and what is morally impermissible and much of what occurs in childhood is moral training, thus Harman's claim is difficult to accept.

Finally, we have grounds for rejecting the poverty of moral stimulus argument due to the fact that moral maturity develops at different ages. Unlike language acquisition, where children usually begin speaking in full and coherent sentences by the age of two and a half, we cannot pin point an exact age where children begin to moralize. Although, as the nativist has pointed out, children are usually able to distinguish between moral and conventional norms by the age of three, it is difficult to make the claim that children begin to moralize at age three, or any other age. Moral norm learning depends on the child's upbringing, exposure to certain environmental stimuli, parental reinforcement, etc. If children are exposed to more or fewer environmental factors that pertain directly to moral acquisition throughout early childhood, they may acquire morality earlier or later than their peers. Unlike language acquisition which normally occurs at the same time for all children, regardless of how little or how much environmental stimuli they are exposed to (although they must be exposed to a specific amount of environmental factors). The same cannot be said of moral acquisition. Thus, we cannot assume that the environment plays a minimal role and is impoverished as is the case with language acquisition. 
Dwyer argues that we obviously need the environment in order to acquire morality, and moral nativists are not denying this. Dwyer claims that simply because the environment plays a role in moral acquisition does not mean we should exclude the contribution of an innate endowment and be content with capacity nativism. In addition, Dwyer argues that if a child has a certain capacity, such as the acquisition of moral behaviour, the child must have access to an environment that makes that possible, and "the logical space encompasses innate endowments and information available in the environment. The nativist does not deny that the environment is relevant; some things must be manifested by adults...but no one gets to exclude by fiat the contribution of an innate endowment." 16

As I have attempted to show in this section, the environment and an innate language faculty play significant roles in language acquisition. If we could prove that language can be acquired solely through environmental factors, then we can exclude the possibility of a language faculty; this is obviously not the case. With moral acquisition, there is reason to believe that our stimuli are not impoverished as they are with language acquisition.

\section{Problems with Dumbfounding}

Susan Dwyer argues that there must be something innate and similar to a language faculty with morality because we experience moral dumbfounding. Dwyer claims that when pressed to explain why we make certain moral judgments, we usually cannot provide a reasonable explanation as to why we judged a certain action as permissible or impermissible, and a similar phenomenon occurs when individuals attempt to explain why we add 'ed' to the end of some past tense verbs but not all. Thus, the LA is a valid analogy when arguing in favour of a moral faculty. 
The problem with Dwyer's argument is that we do not experience a significant amount of dumbfounding in moral decision-making. Rather, we are usually aware of the reasons we deploy when we make a moral judgment. For example, we can probably explain to a judge why we think that a murderer should be incarcerated, in addition to explaining why murder is wrong; we are not dumbfounded in explaining why murder is impermissible. Similarly, when debating on issues with regard to euthanasia, both sides usually have strong arguments for judging euthanasia to be morally right or wrong. For instance, many practitioners may hold the Hippocratic Oath near and dear to their hearts and therefore judge euthanasia as wrong and impermissible, while a son who witnesses a parent in excruciating pain may believe euthanasia is right and permissible under specific circumstances. Thus, although Dwyer argues that we are dumbfounded after making moral judgments, we are usually aware of the reasons we deploy when we make a moral judgment and do not experience the same sort of dumbfounding as we do when attempting to explain why the rules of language are the way they are.

But surely we experience some dumbfounding when we make a moral judgment. For instance, most individuals are dumbfounded when they are keen to explain why incest is wrong and impermissible when the consequences are positive, and when they claim that it is morally right and possibly obligatory to pull the lever to change the direction of the trolley to save the five hikers but morally wrong to push the large man off the bridge. Although dumbfounding is experienced when making specific moral judgments, this does not entail that the dumbfounding experienced is similar to the dumbfounding experienced in language, nor does it entail that moral acquisition is similar to language acquisition. Dumbfounding is a rarity in moral decisionmaking, while with language we are nearly always dumbfounded, unless we are a linguist, in explaining the rules of grammar. Children are always dumbfounded in explaining linguistic 
rules, and this is not the case for morality. If you ask a child why they were put in timeout for hitting their peer, they would probably respond that it is wrong to hit others and they are being punished for behaving badly. In addition and as previously mentioned, moral behaviour is acquired in a very different manner from language, and we usually have supportive reasons as to why we judge some moral situations permissible and others impermissible, while with language we tend not to have supporting reasons but are not conscious of the rules we deploy.

Harman claims that moral reasoning is sensitive to complex principles that most people are not conscious of, and it is unclear how individuals may learn these principles, thus it is plausible that these principles are built in ahead of time in a moral faculty. However, most individuals are aware of the reasoning they employ when deliberating and thinking about moral dilemmas. Churchland also notes that it is likely that moral dilemmas are not addressed instantly or automatically, but reflectively and deliberately, some for extended periods of time. In addition, some moral dilemmas may remain unsettled, and many individuals may struggle long and hard over how to handle a moral problem, as initial intuitions may backfire. ${ }^{17}$ Also, individuals may be unaware that they are employing the doctrine of double effect when they make moral judgments in the trolley dilemmas, but this does not entail that moral principles are built in ahead of time in a moral faculty. Rather, and as previously mentioned, a ToM and a theory of action may be innate capacities that are used in judging moral dilemmas that individuals are not consciously aware of.

Thus, Haidt would be correct in claiming that we experience dumbfounding when judging incest as wrong and impermissible even if there are positive consequences, but incorrect in claiming that our innate purity domain drives our decision. In addition, Dwyer would be correct in claiming that moral dumbfounding is a real phenomenon, but incorrect in claiming that 
moral dumbfounding is similar to the dumbfounding we experience with language. Although we experience moral dumbfounding, moral dumbfounding does not occur often, and we do not need to postulate a moral faculty that is similar to a language faculty to explain moral dumbfounding, as we will further discuss in the following chapter.

\section{Additional Problems with the Linguistic Analogy and PP}

Let us begin this section with looking at the important differences between language acquisition and moral acquisition, where we will continue to discuss and explicate problems with the poverty of moral stimulus argument to understand the problems with PP. First, language is out of sight and out of influence while morality is not. The rules of grammar and syntax that children express when they begin speaking are out of sight and influence, as children are not readily exposed to these rules nor are they taught these rules prior to the age of thirty months, which is when children typically begin speaking in full and coherent sentences. Thus, it would be difficult to make the claim that the environment somehow provides children with enough stimuli to account for language acquisition.

In acquiring morality, children are constantly being punished or given lectures explaining why various actions are right and others wrong. For instance, when a two-year old pushes another two-year old or steals another child's toy, the child's parent will denounce the child's actions and then explain why those actions are wrong. The act of explaining why certain actions are wrong enables a child to learn that pushing and stealing are wrong and are therefore not permissible actions. If a child decides to share their toy with another child, the child's parent will usually respond with a positive attitude, encouraging the child to continue sharing. If a parent continuously responded negatively to sharing and positively to stealing, a child would probably believe that stealing is right and sharing is wrong. In fact, many childhood bullies are probably 
not receiving negative feedback from their parents, where parents discourage bullying and explain why it is wrong. This is not to say that the bully's parents encourage bullying, but they probably do not respond negatively to the action or explain to the child why bullying is wrong.

Peers and educators also play a significant role in shaping a child's morality. Peers and educators will usually respond negatively to a bully's behaviour, thereby isolating the bully from interacting with others. Of course, no child wants to feel isolated and the bully may learn that if they do not bully others, they may be welcomed into the social circle. For example, if a bully pushes another child or steals their toy, the child will probably respond negatively and withdraw from playing with the bully. If the action is witnessed by an educator or parent, they will also respond negatively. All of this educating and associative learning, namely associating an action with a specific reaction from others and learning that negative reactions and reinforcement entails that the action is wrong or not permissible, occurs at the beginning of childhood, namely, when children begin to play with others and begin to exhibit moral behaviour. Thus, while the rules of grammar and syntax are out of sight and influence, where children are not exposed to these rules and violations of language are not treated with negative reinforcement, morality is in sight and is influenced by peers, teachers, and parents, and is treated with negative or positive reinforcement. Much of what goes on in early childhood is training in morality, and there is an enormous amount of socialization in children. Thus, there are grounds for rejecting the nativist claim that children are not exposed to much morality but are able to moralize in the same way that children are not exposed to much language but are able to speak and express the rules of grammar and syntax.

There is also a difference between moral significance and language significance. The shaping of morality and language do not involve similar processes. Language acquisition 
requires much less exposure to the environment and requires much less training than moral acquisition. With language, children do not feel bad if they say that they 'runned to the park' rather than 'ran to the park' because parents and educators do not engage in negative reinforcement when children confuse verb rules. Parents do not respond with a negative emotion or attitude when children misuse words, therefore children do not learn language through associative learning as they do with morality.

Children feel bad when they are punished for a certain action, and they tend to remember what that emotion feels like so they do not engage in that behaviour again, and they often come to have the same dispositions when others engage in that behaviour toward them. Parents and educators treat moral transgressions more seriously and more significantly than they treat transgressions and violations of linguistic rules. Moral acquisition and language acquisition are shaped quite differently, and moral acquisition requires much more training and reinforcement from the environment than language acquisition requires.

Another significant difference between language acquisition and moral acquisition is that there is a critical period for language acquisition, but there may not be a critical period for moral acquisition. ${ }^{18}$ Prinz points out that we are unsure as to whether there is a critical period for morality, but there are reasons to doubt that there is. For instance, children, such as Genie or the wild boy of Aveyron, who were raised in isolation but do not appear to be morally deficient after they are trained to behave morally, and individuals change their moral values throughout their lifetime as well as acquire new moral values. If morality can be acquired after a specific age when children usually begin displaying moral behaviour, and if we can significantly change our moral values throughout our lifetime, there is reason to doubt that after a specific age has passed, morality cannot be acquired. In addition, when comparing two moral systems or when debating a 
moral topic, such as abortion or gun control, individuals assume that their moral system is the correct one. With languages that differ in grammar rules, individuals do not tend to think that one grammar is better than the other or that the other grammar is wrong. ${ }^{19}$

Before turning to counter arguments from the nativist, let us look at PP and determine if PP applies to morality in the same way that it applies to language. Recall that the nativist claims that cross-cultural differences in moral decision-making do not weaken the plausibility of a moral faculty because the environment plays a significant role in shaping moral principles. Although the principles of moral decision-making are innate, the environment determines how those principles are shaped and which norms a culture or group of individuals will endorse. Thus, the principles are innate and the parameters set the constraints on which norms a culture will endorse and promote. Like language, then, the innate rules operate unconsciously and cannot be accessed, but guides moral decision-making and how we treat moral transgressions.

However, there is a significant amount of difference between variation in language and variation in morality. With language, there is a fixed amount of variation and the differences are not significant. For instance, there are only a specific number of languages that have been produced to date, and all languages are similar, such as Hindi and Urdu or Spanish and French. In addition, the subject-object-verb order differs among languages but, while languages are subject-object-verb or subject-verb-object ordered, no human languages are object-subject-verb ordered. Despite the differences, all humans begin speaking at the same time and with little help from the environment, and the variation among languages is limited and specific.

In comparing the moral faculty to the language faculty, moral nativists argue that the same rules apply. They claim that the variation among moral norms are limited and specific, arguing that some moral norms can never be generated, and the differences across cultures in 
making moral judgments are not significant. However, we have already seen that there is in fact a significant amount of difference in the way separate cultures make moral decisions and the variation is not fixed as it is with language. There is a significant amount of variation between cultures in their treatment of harm, sharing, and incest norms. If humans are endowed with a moral faculty or innate foundations of morality, as the moral nativist argues, there should not be a significant amount of variation in moral decision-making, but limited and specific variation. However, the variation is not limited and specific, and cultures do vary significantly in the way they make moral judgments to the extent that certain norms may not appear universal, even at a very general level. If the moral nativist is correct in claiming that we have a moral faculty that is similar to the language faculty, then there should not be a significant amount of variation in moral decision-making.

Hauser claims that the language faculty is a complex faculty and requires that the environmental input interact with the innate structure and maturational factors in order to develop properly. He claims that

while it is obvious that much of language is learned--for instance the arbitrary mapping between sound and concept--what is less obvious is that the learning of language is only possible if the learner is permitted to make certain initial assumptions. This boils down to a question of the child's initial state--of her unconscious knowledge of linguistic principles prior to exposure to a spoken or signed language. It has to be the case that some innate structure is in place to guide the growth of a particular language. ${ }^{20}$ In comparing the moral faculty to the language faculty, Hauser claims that although reasoning, emotions, and other capacities in the human mind play a role in making moral judgments, the moral faculty is comprised of unconscious or operative knowledge of moral principles prior to 
exposure of morality, and variation in moral decision-making is based "on how each culture switches on or off particular parameters. An individual's moral grammar enables him to unconsciously generate a limitless range of moral judgments within the native culture."

However, and as previously mentioned, linguistic rules are parameterized and the language faculty has a small set of possible settings that are triggered by the environment and experience, but the same cannot be said about morality. For instance, while there are a small set of possible settings that can be triggered in language, it is unclear what the parameters are supposed to be for morality. ${ }^{22}$ With language, the linguistic parameter settings correspond to the structure of the language faculty and there is not a significant amount of variation; the same cannot be said about morality. Conservatives and liberals have conflicting values, as Haidt has pointed out. However, while Haidt claims that these values are different parameter settings on the same basic set of innate rules or principles, conservatives and liberals may simply have fundamentally different principles guiding their values. Simply, there is too much variation to suggest that rigid parameters are responsible for shaping these values, and moral values do not seem to be structured in the same way language is structured, where variation corresponds to the structure of the faculty. In addition, there are conflicting values and debates about moral judgments, such as debates about gender equality, capital punishment, and gun control, and these conflicting values cannot be treated as parametric variations, unless we trivialize the idea, suggesting that each conflicting or contested policy is a parameter in its own right that can be switched off or on, which reduces the plausibility of the LA. ${ }^{23}$ Conservatives and liberals may simply have fundamentally different principles, in the same way that different religions or different cultures have fundamentally different principles. Thus, values and moral judgments are open-ended rather than parametric. 
In an attempt to save the LA, Dwyer claims that the LA is useful when arguing in favour of a moral faculty due to the fact that certain moral norms will simply never be generated in the same way that some languages will never be generated, and this constraint occurs because of our innate moral faculty. As previously mentioned, moral nativists argue that while there is moral variation which can be attributed to the social environment, there are some rules that no moral creature could generate (e.g., let's torture babies for fun). With language, we see the same kind of variation, although there is of course much less variation. However, moral variation is openended (e.g., Nazis inculcating hatred toward the "non-ideal" race, Aztecs valuing cannibalism, etc.) while variation in language is closed. Although moral nativists attempt to compare the moral faculty to the language faculty, the LA is not useful and morality is not constrained in the same way that language is constrained. Thus, it appears that the LA weakens the moral nativist position by emphasizing the significant differences and disanologies between language acquisition and moral acquisition rather than lending support to moral nativism.

\section{Universality Does Not Require Innateness}

Let us examine the problems associated with the nativist argument of universality requiring or entailing innateness. We will look at the problems with making a leap from universality to innateness, while at the same time looking at the argument that there may be no universals in moral decision-making. More specifically, I argue that there are no universal norms as nativists believe, and even if there are universals in moral decision-making, that does not entail or require innateness.

As mentioned in the previous chapter, nativists argue that there is quite a bit of universality in moral decision-making despite cross-cultural differences. Hauser argues that although we do in fact witness variation with many harm norms, such as prohibitions against 
killing, each culture or society will set the boundaries or parameters which determine when killing may be permissible. He argues that killing may be permissible in many instances, such as war, intolerable pain, etc. However, the harm norm that prohibits killing is a universal norm that functions at a very general level, and this universal norm is a product of our moral faculty. Dwyer and Haidt maintain that certain norms cannot and never will be generated, such as torturing babies for fun is good, and this constraint is also a product of the moral faculty. The moral nativist thus maintains that universality is a product of our moral faculty and occurs because all humans are endowed with a moral faculty.

There are a few problems with this view. For starters, despite the fact that there is some universality in moral decision-making, there are many differences across cultures that cannot be accounted for via a universal moral grammar. For example, Hauser claims that harm norms are universal, and Haidt argues that incest norms are also universal. However, if harm norms and incest norms are both innate and universal across cultures, we would not witness the exceptions to killing or variations on which relationships count as incestuous. In other words, there would not be a significant amount of variation across cultures in moral decision-making. The fact that there are harm norms in place in every culture is in fact a feature of human morality. Thus far, there has not been a record of a culture that does not have some form of a harm prohibition. However, one would be mistaken to argue that this feature of morality is a product of a moral faculty due to the fact that human brains are similarly organized, and humans will therefore devise similar solutions to the same problems. Hauser, Dwyer, and Haidt argue that harm norms are universal across cultures and universality would not occur without a moral faculty or an innate foundation of harm. However, simply because there are general themes of prohibitions against harm does not entail that there are universal norms or that humans have an innate moral 
faculty prohibiting certain types of harm. As Churchland points out "similarly organized brains facing similar problems are likely to land on similar solutions. ${ }^{, 24}$

Biologically speaking, it is not contested that there are numerous similarities between individuals, irrespective of location. Because humans are designed in a similar fashion and because humans have probably faced similar issues and continue to face similar issues, individuals may respond in similar manners. However, we do not need to postulate a moral faculty to explain these similarities, rather, we can explain these similarities by appealing to the fact that each human brain is similarly organized, thus different societies may have similar solutions to the same problems. For example, fire-making and marriage are common themes across cultures, but it would be rather odd to suggest that we have a fire-making or marriage faculty. Rather, different cultural groups tend to face similar problems, and because our brains are similarly organized, we may come up with similar solutions. As Churchland notes, "wood works well in boatbuilding, merriment eases social tensions, and competitive games are less costly than fights. ${ }^{, 25}$ These are all similar and common themes across cultures, and because humans are biologically structured in similar ways, it entails that there will be many common themes across cultures. However, postulating an innate faculty or module for every common theme would be rather extravagant.

In short, harm norms may simply be social constructs that were created in order to maintain stable societies. We will further discuss this possibility in the following chapter. There are also significant differences in harm norms across cultures, so much so that although harm norms are a feature of human morality, they may not be universal in the way moral nativists believe them to be, as we will see later on in the chapter. 
Another point to consider is that if moral decision-making is both universal and innate, then cultural differences would not be so divergent. Rather, the rigid parameters would set a boundary as to how much diversity can occur in moral decision-making across cultures and within cultures, as there is also variation in moral decision-making between individuals who reside in the same society (e.g., moral judgments on capital punishment). Churchland asks us to consider one of Hauser's examples in favour of a moral faculty, where Hauser claims that just about every subject who filled out his questionnaire responds in a disgusted manner when confronted with the idea of drinking apple juice out of a brand-new hospital bedpan. Churchland claims that of course most individuals would not find this idea appealing when sitting at a desk and responding to this question simply because bedpans are associated with urine, thus we obviously would not want to drink apple juice out of a bedpan. However, if an individual were in a different context, such as desperately dehydrated in a desert, they probably would not find the idea of drinking apple juice out of a bedpan unappealing or disgusting. ${ }^{26}$ Thus, if moral nativists are going to argue that universality is a product of our moral faculty, or that there is universality in moral decision-making, they need to account for variation in moral decision-making across different contexts, not just across cultures and between individuals. It is also important to note that although Hauser claims that this situation is moral, it is unclear whether it counts as a moral dilemma and I am dubious about considering it moral.

The main point here is that universality, if there is a case for universality in moral decision-making, which can be contested at this point, is consistent with the existence of an innate moral faculty or module. However, it does not imply the existence of such a mechanism, and moral nativists need to supply compelling evidence in addition to universality. ${ }^{27}$ 
Recall that Dwyer claims that regardless of environmental factors and moral training in early childhood, there are certain moral judgments that can never be generated, such as torturing babies is fun, and this is a universal feature that is a product of our moral faculty ${ }^{28}$ The problem with Dwyer's claim is that unfortunately, certain moral judgments, such as torturing babies is fun or claims such as certain races should be exterminated because they are impure, can and may be generated, and history is sufficient in proving Dwyer's claim wrong; as Prinz notes, "unlike language, where certain grammars apparently never occur, moral variation seems to be relatively open-ended. ${ }^{29}$ For instance, in Ancient Greece infanticide was common and not considered immoral, scarification is common in many societies today, the Romans were fans of blood sports, the Aztecs were cannibals, and so on. ${ }^{30}$ As Prinz puts it, "torture comes all too easily."31 While moral nativists compare the moral faculty to the language faculty, thus invoking a Principles and Parameters component, the constraints for language are much stronger than they are for morality. Essentially, the environment plays a much larger role in shaping moral acquisition than it does in shaping language acquisition, and we have seen throughout history how the social environment, education, and reinforcement from parents, teachers, and peers are the primary contributors of moral acquisition. Of course, this does not entail that any moral norm will be generated simply because "nonmoral preferences (such as parental instincts) and the need for group cohesion may render certain moral values unlikely [sic], ${ }^{, 32}$ and we do not need to postulate a moral faculty for these constraints.

Prinz argues that in order to use universals to argue for innateness, a moral nativist should prove the following: first, that there are in fact moral universals, and second, there are not any plausible nonnativist explanations for these universals. ${ }^{33}$ As previously discussed, there are very general universal moral norms, such as prohibitions against harm and torture. However, the 
specific content of these universal harm norms differs greatly across cultures, and there appears to be "massive cultural variation in who can be harmed and when. Within our own geographic boundaries, subcultures disagree about whether capital punishment, spanking, and violent sports are permissible. Globally, every extreme can be found., ${ }^{, 34}$ It is important to remember that the moral nativist is not arguing that there are not differences across cultures, but arguing that the differences occur due to the process of environmental factors shaping the moral faculty. However, variation in moral decision-making, especially with regard to harm norms, differs significantly and therefore cannot be adequately explained by a PP approach to morality. As we have seen throughout history, "the anthropological record suggests that the range of overriding factors is open-ended. We can harm people for punishment, for beauty, for conquest, and for fun." ${ }^{\text {35 }}$ According to Prinz, harm avoidance may not fall under the category of a universal feature of moral decision-making and may not even be considered a universal moral imperative because of the significant amount of variation witnessed throughout history and between cultures. ${ }^{36}$

Due to the fact that universality in harm prohibitions occurs at such a general level, Prinz claims that harm prohibitions are not universal in form and can be explained without postulating an innate moral faculty. Rather, we can account for harm prohibitions by appealing to the fact that we may be biologically prone to caring about one another ${ }^{37}$ Similarly, Churchland notes that trust, cooperation, caring, and oxytocin are significant factors in moral decision-making that account for human morality and we do not need a moral faculty to explain moral decisionmaking. ${ }^{38}$ In addition, Prinz claims that "there are universal constraints on stable societies, which tend to promote the construction of rules against harm...other species don't go around killing each other constantly, but we are not tempted to say that they have moral rules against harm." ${ }^{, 39}$ Thus, lacking an innate moral faculty that prohibits harm does not entail that humans will be 
nasty and vicious toward one another. Rather, the need and desire for stable and safe societies will drive the construction of harm norms without the assistance of a moral faculty.

Moral nativists also claim that norms of sharing and reciprocity are both universal and innate. They claim that like harm norms, norms of sharing and reciprocity tend to be similar across cultures although there is variation on how each culture sets the parameters of these norms. Sure, it is the case humans sometimes tend to share and reciprocate. However, the way in which cultures share may vary significantly, thus a universal component of sharing as well as an innate principle that guides sharing and reciprocity can be called into question.

Despite the fact that most societies invoke some form of a sharing norm, societies and cultures vary significantly in the way they share. For example, in our culture, the wealthy are expected to pay significant taxes but are not expected to divide their profits. In Bolivia, sharing is not common or expected, and in some cultures men are not expected to share meals with women and children. ${ }^{40}$ What is more, anthropologists note that there are significant differences in the way people share across cultures. In addition, most individuals tend to share only within their own social group or community and exclude sharing with out-groups and distant people, as we have previously seen.

When anthropologists conducted a series of studies that focused on conceptions of sharing in fifteen small-scale societies, they found that societies have very different conceptions of what 'fair' and 'sharing' are. Anthropologists presented subjects in these societies with the ultimatum game. There are two players in the ultimatum game. Player A is given a certain amount of money, say, ten dollars. Player $A$ is told that he can keep some of the money for himself and share some with the second player, Player B, who is not related to Player A. If Player B does not accept what Player A offers, both players leave with nothing. Therefore, 
Player A is motivated to make a fair offer that they think Player B will accept because if Player B considers the offer unfair, he will reject the offer and each player receives nothing. Thus, it is in Player A's interest to offer a fair amount.

According to the studies, players in the West tend to offer forty-five percent of the money. On the nativist view, offering forty-five percent of the money occurs because our moral faculty guides the decision to share. However, we probably offer that much out of self-interest because we would prefer to keep some of the money rather than none. When tested in smaller scale societies, players offer considerably more than one percent. However, while players in the West offer forty-five percent on average, in other cultures some players offer a little more while some offer considerably less. In Peru, players in the Machiguenga culture offer twenty-six percent on average, and the most frequent offer was fifteen percent. ${ }^{41}$ If players in the West offered fifteen percent, most individuals would find it unfair and reject the offer, probably out of spite. It appears, then, that the Machiguenga in Peru have a different standard of sharing. While they may have a sharing norm, the way they share varies significantly from the way we share. Thus, although the moral nativist argues that sharing is an innate component of the moral faculty, the amount we share with others is variable. In ultimatum games, there are considerable differences in terms of sharing and reciprocity across cultures which are sufficient to dismiss the claim that humans are endowed with an innate sharing principle that produces a universal sharing norm. Again, while most cultures do in fact have sharing norms, they are not universal in the way that moral nativists believe them to be, as there is a significant amount of variation in sharing norms across cultures. There is simply too much variability in sharing norms for sharing norms to be considered universal. In Chapter III, we will look at a nonnativist argument that sufficiently and adequately explains the differences in sharing norms across cultures, as well as 
explaining why many individuals tend to share with one another even in the absence of immediate benefits.

Finally, although I have previously touched on this topic briefly, let us further examine the case for universality in incest norms. Moral nativists argue that most individuals believe that incest is morally wrong, irrespective of location. In fact, Haidt notes that even if the act of incest is somehow beneficial or positive rather than negative, most individuals maintain that incest is wrong. In addition, Prinz notes that just about every culture thus far studied condemns incest. ${ }^{42}$ According to the nativist there must be an innate principle or moral foundation prohibiting incest. However, there is a significant amount of cultural variation with regard to which relationships count as incestuous. In many cultures, incest is restricted to immediate family, such as siblings and parents. In other cultures, incest includes first and second cousins, while in others incestuous relationships include sexual relations with a childhood friend. In addition, the incest taboo has changed throughout history. For instance, "the medieval Church prohibited marriage with cousins up to seven degrees...in the ancient world, cousin marriage was commonplace., ${ }^{, 43}$ Moreover, marriages between first cousins in Saudi Arabia and Pakistan seem to be quite common. ${ }^{44}$ Thus, we do not have reason to believe that there is an innate taboo or rule against incest outside of the immediate family, but we may have reason to believe that there is a universal norm against incestuous relationships with immediate family members, although again, simply because incest norms may be universal does not entail that we have an innate foundation prohibiting incest. In Chapter III, we will turn to an explanation given by Prinz that accounts for incest norms without postulating a moral faculty.

The most common nativist counter objection to these objections is that cultures determine the scope of moral principles. Moral nativists, such as Hauser and Dwyer, argue that significant 
variation in moral decision-making is due to the environment's role in shaping the innate moral principles. They maintain that the vast and significant variation in harm and fairness norms across cultures does not pose a threat to moral nativism, rather, it fits well with moral nativism and PP. As we have seen earlier on in this chapter, there are many difficulties facing PP and moral nativism. If the argument from universality, the fourth main argument offered for moral nativism, is also weak, we have reasons to dismiss moral nativism.

\section{Problems with SIM}

In this section I look at the features that are common to the nonnativist accounts and Haidt's model, and look at what a nonnativist about morality would accept and reject in Haidt's model, emphasizing the commonalities between Haidt's SIM and nonnativist theories of moral acquisition. Although nonnativist theories will be discussed in the following chapter, I have discussed some components of nonnativist theories in this chapter as well as in Chapter I, and will therefore focus on the components of nonnativism previously discussed.

There is not much that a nonnativist would not accept of Haidt's SIM if Haidt were to dismiss innate foundations or domains of moral decision-making. A significant similarity between the SIM and nonnativist theories such as those proposed by Sripada and Prinz is the importance of emotions. Both Prinz and Sripada emphasize the importance of specific emotions and the role emotions play in moral decision-making. For instance, both philosophers agree that disgust plays an important role in making the moral judgment that incest is wrong. In addition, both Prinz and Sripada agree that disgust is an innate emotion and is elicited under certain circumstances and may or may not be moralized. Prinz points out that disgust, or any other emotion, can play a role in moralizing only if a rule is enforced by the emotion of self-blame and other blame and is directed at a third party. ${ }^{45}$ Recall that according to Prinz, children learn moral 
norms through associative learning. Children associate specific reactions from parents and educators, such as negative reinforcement or a negative attitude, with norms that are impermissible, and thus associate negative reactions from an adult or educator with self-directed guilt or contempt for having performed that action, and will also want others to feel guilty or angry when they perform a similar action. Sripada also reinforces the role of emotions when he claims that specific emotions, such as disgust, serves as the origin and maintenance of specific moral norms, such as a norm against incest or eating meat in certain cultures. However, if an emotion is not moralized and if a specific action does not receive negative reinforcement and a negative attitude despite eliciting a specific emotion, chances are that action will not be turned into a moral norm prohibiting the action. For example, we may feel sympathy for a sick individual, but we do not make moral judgments about them, or we may feel disgusted at someone who eats a significant amount of tripe or who eats more than they should, but in our culture we would not claim that they are morally wrong for doing so.

Haidt also argues that emotions play a significant role in moral decision-making. Recall from Chapter I that Haidt argues that individuals are morally dumbfounded when they make moral judgments because they are guided by their innate moral foundations, such as harm or purity, and these foundations are formed from specific emotions, such as sympathy or disgust. However, Haidt diverges from the nonnativist when he argues that the five innate foundations of moral decision-making take the form of Sperberian modules. Recall from the Introduction that Sperberian modularity is the mediate position between Fodorian modularity and massive modularity. According to Haidt, the five foundations of moral decision-making are innate in all individuals, and guide moral decision-making. 
One of the problems with this view is that some of the five principles, such as community and authority, run counter to our conceptions of morality. For example, the community principle entails that we evolved with a bias toward helping others who are near and dear over helping others that are outside of our community. According to the community principle, we tend to invoke sympathetic feelings for "insiders" rather than "outsiders", unfortunate as this is. According to this line of reasoning, natural selection propagated a community principle in humans, where the ability to sympathize with individuals in our own community is more likely to occur when they stumble upon some form of misfortune, than sympathizing with individuals who are a great distance from us who may be in a worse position. This line of reasoning can be found in a well-known ethical dilemma, previously discussed in Chapter I (see page 15): you are driving in your new fancy car with leather seats. You see a wounded individual on the road who asks you to give him a lift to the hospital. His leg is badly wounded and he is losing a lot of blood, and without medical attention in the next two hours, he may lose his leg. However, his blood will get all over your leather seats and they will need to be replaced at a cost of two hundred dollars. Do you take him to the hospital? Most individuals would say yes. Consider a similar dilemma: You are at home with a delicious bowl of popcorn watching a movie on CTV. A commercial from Children's Fund is aired, and asks you for a small donation of a dollar a day to ensure clean drinking water which will save a child's life. Do you pick up the phone and call to save a life? Most do not, even though this request imposes a smaller cost on an individual and has greater benefits than the former situation.

If this is the case, then some of the five foundations of (so-called) morality may work against their intended purpose. More specifically, if the five foundations of morality guide moral decision-making in a way where we act in a moral manner and consider specific actions immoral 
or moral, then our moral judgments should not be counterintuitive. For instance, we have an intuition that incest and murder are wrong and that pulling the lever to redirect the trolley in order to save five lives is right, but we do not have an intuition that donating to individuals outside our community is obligatory in the same way it might be obligatory to assist individuals in our own community. Thus, we have reason to doubt that all five foundations of morality are moral in the way Haidt believes them to be. Although Haidt does recognize that the five foundations of morality can be counterintuitive, he insists that the foundations are moral foundations and forms the basis of moral decision-making. Haidt argues that the foundations are moral foundations because without these innate foundations, acquiring moral behaviour and making moral decisions would not be possible. Haidt claims that "all cultures are constrained by the five foundations of intuitive ethics, ${ }^{, 46}$ namely, the five foundations of morality, so much so that human morality would not be possible without it.

It is important to note that when nativists argue in favour of innate moral foundations or a moral faculty, two possibilities arise: first, due to evolutionary factors, natural selection selected a moral faculty or innate moral foundations, where humans were selected to act in a moral manner. Second, natural selection selected components of behaviour, such as the five moral foundations, in order to ensure survival and stable fitness levels and did not select these principles in order to produce moral behaviour. If this is the case, these components would have eventually worked together to produce what we now define as moral principles and moral behaviour, despite the possibility that these principles were not selected in order to produce moral behaviour. Thus, the moral faculty may be an exaptation and may not actually be 'moral' in the sense moral nativists advocate, therefore we may have grounds for rejecting a moral faculty and endorsing the view that morality may be a by-product of other faculties. 
The problems with Haidt's supposition are first, these domains of decision-making may be universal as Haidt claims but may not necessarily be moral; second, although Haidt's SIM allows for more flexibility in moral decision-making than Hauser and Dwyer's theories, there is too much variation in moral decision-making for this model to be considered plausible. For example, there is reason to believe that some domains are not construed as morally significant in some cultures, although they may be construed as morally significant in others; and third, some of the principles, such as purity, may simply be emotion-based (e.g., the emotion of disgust may favour norms against incest and meat-eating) which does not entail that the principle is innate, though the emotion may be innate. Let us examine each of these criticisms in turn.

In accord with the previous section of this chapter, Haidt argues that the five domains of morality are universal and guide moral decision-making, which explains universality in moral decision-making and accounts for cross-cultural differences. Haidt argues that some cultures may emphasize some of the domains more than others and culture plays an important role in shaping the innate domains, therefore cross-cultural differences are inevitable. For instance, the purity domain may be emphasized to a greater degree in low-socioeconomic-status (SES) populations in Brazil, as individuals in this culture believe that a man who masturbates in a chicken carcass and then cooks it for dinner is immoral, while high SES American college students find this act disgusting but not immoral. The harm domain is also emphasized to different degrees in different cultures. For example, the Gahuku Gama head hunters in Papua New Guinea do not consider harming members outside of their group immoral, but only immoral when a member of their own group is harmed. Thus, in the Gahuku Gama society the harm foundation is not treated as being as important as the hierarchy and reciprocity foundations. ${ }^{47}$ Therefore, according to Haidt's SIM, 
because every culture emphasizes each domain to a greater or lesser degree, we witness crosscultural differences in moral decision-making.

Let us return to Haidt's example of the man who masturbates in a chicken carcass and then cooks it for dinner. Recall that low SES individuals in Brazil find this act immoral while American college students find it disgusting but not immoral. Haidt argues that this difference in moral decision-making occurs because the purity domain is emphasized to a greater degree in low SES Brazilian societies than in middle-class American societies. However, this does not entail that the purity domain, or any of the other four domains, are moral. They may be universal, but they may not necessarily be moral. I am not contesting the fact that certain emotions are innate, such as sympathy, disgust, anger, etc., but challenging the claim that the domains of moral decision-making are moral. The innate emotion, such as disgust, may explain how certain purity domains emerge over time, and explains why some cultures moralize the emotion while others do not. For example, many things are disgusting but we do not consider all disgusting acts or things immoral, such as rotting meat, pollution, bodily fluids, eating meat, spitting, oral sex, etc. ${ }^{48}$ We find some or all of these behaviours or things disgusting simply because we have natural disgust elicitors. While Haidt argues that the purity domain is the foundation of morality and moral norms, it may be the case that natural disgust elicitors form the basis of our moral judgments.

In order for a behaviour, such as eating meat, to be considered immoral, a society must draw attention to the similarity between eating meat and the natural disgust elicitor, and it will elicit feelings of both self-and other-directed blame toward individuals who eat meat. Thus, "once a society uses disgust to moralize certain behaviours, its members can be said to have a purity domain in their moral psychology. ${ }^{, 49}$ If this view is plausible, then the purity domain can 
be viewed as a learned or socialized extension of the emotion disgust, and the same reasoning applies to the other four domains of moral decision-making. For instance, the fact that we feel anger toward a murderer and sympathy for the victim does not entail that we have an innate harm principle. The harm domain may be an extension of these innate emotions. According to this theory, the harm domain may be an extension of the following: if we engaged in an act of harm toward another individual, we would probably feel guilty or angry, or possibly even disgusted at ourselves after having harmed someone, and we would also want others to feel just as blameworthy if they acted in a similar manner. According to Prinz, "anger and disgust toward others take on a moral cast only when we would feel blameworthy ourselves for behaving in a similar action." ${ }^{50}$ Although this may not always be the case, according to this line of reasoning the domains might be universal in the sense that all cultures express these domains, however, they may not necessarily be moral and may be a learned extension of our innate emotions. The point here is that while the domains that Haidt argues for may be universal and possibly innate, they may not necessarily be the foundations of morality.

This leads us to another problem with Haidt's SIM. While Haidt's SIM allows for more flexibility in moral decision-making than Hauser and Dwyer's theories, there is too much variation in moral decision-making, therefore there is reason to believe that some domains are not construed as morally significant in some cultures although they may be construed as morally significant in others. Essentially, the domains may not be foundations of morality, as already mentioned. As previously discussed, some cultures may not view some domains as morally significant while other cultures may emphasize the domains to a significant degree. Although Haidt does allow for variation in moral decision-making, claiming that differences can be attributed to the environment and how cultures treat the importance of each domain, Haidt does 
not take into account that some cultures simply may not moralize a specific emotion that is particular to a moral domain (e.g., disgust is particular to the purity domain), thus there is reason to believe that the domains are learned domains, as previously discussed, and may not actually be moral although they may be universal. Therefore, there are reasons to reject Haidt's argument that the domains are construed as morally significant in every culture although some cultures may emphasize each domain to a greater or lesser degree than other cultures. Thus, the domains do not play the same roles across cultures ${ }^{51}$ which entails that we may have innate emotions that can be moralized, but not innate moral foundations or domains of moral decision-making.

\section{Conclusion}

Throughout the course of this chapter, I have critically examined the plausibility of moral nativism. In particular, I have shown why it is implausible to argue that our stimuli are impoverished with regard to moral acquisition, although it may be impoverished with regard to language acquisition. I have also attempted to show why dumbfounding is not a real phenomenon and why PP is not a valid analogy. Lastly, I have shown why universality does not require innateness, and why Haidt's SIM is problematic, emphasizing the importance of moral emotions while rejecting the conception of innate moral foundations. In the following chapter, I put forth alternatives to moral nativism, and show that nonnativist theories are less problematic and more plausible than the nativist theories put forth in Chapter I.

\section{Notes}

\footnotetext{
${ }^{1}$ Mikhail, "Comments on Sripada", 354.

${ }^{2}$ By associative learning, I simply mean learning that takes place through means of associating a specific action with an emotional reaction. For more, see Prinz, 2008.

${ }^{3}$ Mikhail, 355.

"Sripada, "Reply to Harman and Mikhail", 365.

${ }^{5}$ Hauser, Moral Minds, 178.
} 
${ }^{6}$ Hauser, 182.

${ }^{7}$ Hauser, 181.

${ }^{8}$ Ibid.

${ }^{9}$ Prinz, 431.

${ }^{10}$ Ibid.

${ }^{11}$ Prinz, 431, 432.

${ }^{12}$ Shaun Nichols, Sentimental Rules (New York: Oxford University Press, 2004), 28, 128.

${ }^{13}$ Sripada, "Nativism and Moral Psychology", 328.

${ }^{14}$ Ibid.

${ }^{15}$ Gilbert Harman, "Using a Linguistic Analogy to Study Morality" as in article in Walter Sinnott-Armstrong, Moral Psychology: The Evolution of Morality: Adaptations and Innateness vol. 1 (Cambridge: MIT Press, 2008), 349.

${ }^{16}$ Dwyer, 415.

${ }^{17}$ Churchland, 111.

${ }^{18}$ See Pinker, The Language Instinct, 1997.

${ }^{19}$ Jesse J. Prinz, "Resisting the Linguistic Analogy: A Commentary on Hauser, Young, and Cushman" as an article n Walter Sinnott-Armstrong, Moral Psychology" The Cognitive Science of Morality: Intuition and Diversity vol. 2

(Cambridge: MIT Press, 2008), 159.

${ }^{20}$ Hauser et al., 111.

${ }^{21}$ Hauser et al., 118.

${ }^{22}$ Prinz, 159.

${ }^{23}$ Ibid.

${ }^{24}$ Churchland, 103.

${ }^{25}$ Ibid.

${ }^{26}$ Churchland, 110.

${ }^{27}$ Churchland, 108.

${ }^{28}$ Susan Dwyer, "How Not to Argue that Morality Isn't Innate: Comments on Prinz," 414.

${ }^{29}$ Jesse J. Prinz, "Reply to Dwyer and Tiberius" as in article in Walter Sinnott-Armstrong, Moral Psychology: The Evolution of Morality: Adaptations and Innateness vol. 1 (Cambridge: MIT Press, 2008), 429.

${ }^{30}$ Ibid.

31 lbid.

32 Ibid.

${ }^{33}$ Prinz, "Is Morality Innate?", 372.

${ }^{34}$ Prinz, 373.

${ }^{35}$ Prinz, 374.

${ }^{36} \mathrm{Ibid}$.

${ }^{37}$ Prinz, 375.

${ }^{38}$ See Churchland, 2011.

${ }^{39}$ Ibid.

${ }^{40}$ Prinz, 376.

${ }^{41} \mathrm{lbid}$.

${ }^{42}$ Prinz, 378.

${ }^{43} \mathrm{lbid}$.

${ }^{44} \mathrm{Ibid}$.

${ }^{45}$ Prinz, 386.

${ }^{46}$ Haidt and Bjorklund, 213.

${ }^{47}$ Prinz, 382.

${ }^{48}$ Ibid.

${ }^{49}$ Ibid.

${ }^{50}$ Prinz, 383.

${ }^{51}$ Prinz, 381. 


\section{Chapter III: Introduction}

I see no reasonable alternative to the position that grammars are internally represented in the mind, and that the basic reason why knowledge of language comes to be shared in a suitably idealized population (and partially shared in actual populations) is that its members share a rich initial state, hence develop similar states of knowledge. ${ }^{1}$

Chomsky's statement emphasizes that a theory should be accepted only if it provides the best explanation for the data presented, and if there are not any feasible and more plausible alternatives available. In Chapter II we looked at problems with moral nativism and moral nativist theories. This chapter will explore whether the alternatives to moral nativism are more plausible than moral nativism, or whether we have no reasonable alternative to the position that morality is innately represented in the mind. In particular, I will examine Sripada's Innate Biases Model, a theory that shares commonalities with moral nativism but is not nativist itself, Dennett's theory of memes, where, drawing on Dennett's work, I suggest that memes and cultural evolution can explain the emergence, transmission, and maintenance of moral norms, and Prinz's Emotions Constitution Model (ECM), a theory that departs significantly from nativism. I begin with the Innate Biases Model, follow with Dennett's theory of memetics, and conclude with the ECM because each theory is further away from nativism than the previous theory, and all three theories make weaker commitments than nativism and adequately explain the acquisition of moral behaviour. Adopting Occam's razor, which is the thesis that commits to the idea that when comparing theories, the theory that makes the weakest commitments should be endorsed, it will become clear that each theory can account for moral acquisition and makes weaker commitments than moral nativism, therefore we have grounds for endorsing 
nonnativism. Occam's razor states that we should endorse the theory that makes weaker commitments, even when both theories offer plausible explanations. In addition, and this is important for our analysis of moral nativism, adopting Occam's razor entails that we should not endorse the theory that needs to make additional commitments, when the other theory can just as plausibly, if not better, account for the questions being presented. Because parts of these theories have been elaborated in Chapter II, I will focus on what I believe to be the most significant parts of these theories for our present discussion. It is important to note that I am not endorsing any of the three nonnativist theories presented in this chapter. Rather, my aim is to show that all three nonnativist theories plausibly account for moral acquisition and moral norms, and do so in a less complex manner, making weaker commitments than moral nativism, therefore we have reason to endorse nonnativism over moral nativism.

\section{The Innate Biases Model}

Let us begin by discussing the Innate Biases Model, a model that is more specific and complex than capacity nativism, but does not endorse the theory of innate moral principles or foundations. Recall that capacity nativism claims that humans are born with the capacity to act in a moral manner; namely, all humans have innate capacities (e.g., a theory of action) that allow them to act in a moral manner and acquire moral behaviour. The Innate Biases Model proposes that there is more to acquiring moral behaviour than capacity nativism, but does not make the strong commitments that nativism makes. The Innate Biases Model endorses capacity nativism, and argues that in addition to having certain capacities that assist in the acquisition of moral behaviour, the human brain is also endowed with innate biases (e.g., disgust when thinking of incest, repulsion when thinking of harm) that assist in the emergence of moral norms and the 
acquisition of moral behaviour. In this section, I will show that the Innate Biases Model can adequately account for moral acquisition and changes in moral norms throughout history.

The Innate Biases Model proposes that the innate biases that were selected for in humans by natural selection account for the emergence of moral norms. According to Sripada, the widespread presence of certain norms, such as incest prohibitions, can be explained by the fact that the innate structure of the mind, namely certain biases that humans are biologically built with, make us predisposed to favour or disfavour the emergence or propagation of these norms. Innate biases form norms and these norms are transmitted from one individual to another individual or a group of individuals if the normative rules are attractive or accord with one's psychology and emotions. Due to the fact that our brains are similarly organized, a group of individuals may find a set of normative rules attractive because it accords with their psychology. For instance, a group of individuals who are very religious may find the norm prohibiting samesex marriages permissible, while others find it impermissible. Those who wish to prohibit samesex marriages and view it as morally wrong on the basis of religion may have an innate bias of disgust based on impurity when thinking about same-sex marriages. This bias is what leads to the judgment that same-sex marriages are wrong. Alternatively, individuals who find same-sex marriages impermissible may not have any innate biases that form their judgment, rather, they may strongly believe in listening to authority, in this case, the word of God.

Similarly, Shaun Nichols argues that biases, such as disgust, play a role in the transmission and maintenance of moral norms. In Sentimental Rules, Nichols shows that affective responses play a significant role in treating certain disgust violations as immoral rather than morally neutral. If a norm is backed by an affective response, the norm will be maintained and survive. For instance, Nichols shows that certain etiquette norms that were around in the 
Medieval era that were not continuously backed by an affective response are no longer around, while those that were backed by an affective response are still around in contemporary etiquette. The same line of reasoning applies to other norms, such as harm norms. Similarly, in order for an individual to acquire a set of moral norms and moral behaviour, they should have affect-backedresponses to moral situations. Nichols argues that "if the affective mechanism is absent, or, perhaps, if it is absent during some critical developmental period, then the child would not develop a proper nonconventional normative theory." ${ }^{2}$ However, this is not to say that any norm that is not affectively backed will not survive, but the chance of survival is greater if the norm is affectively backed. For instance, many meat-eating prohibitions in Islamic and Hindu cultures are not backed by affect, but by authority. Again, a norm may be backed by something other than affect, such as religious authority, but having an affect-backed-response increases the chances of survival.

Innate biases may also work to create moral norms and may also explain variation in moral norms across cultures. According to Sripada, the Westermarck mechanism, a mechanism that creates an aversion to incest, can explain the emergence and maintenance of incest norms. Edward Westermarck, a late nineteenth century Finnish sociologist, proposed that humans have an innate mechanism that generates a strong aversion to procreating with individuals in the same family group or individuals that spent their childhood years together. Due to the fact that procreation with close relatives cause genetic defects, a mechanism that generates an aversion to procreating with a family member would have been favoured by natural selection. The innate aversion, namely the bias of disgust that prohibits incest, is not an innate moral norm. Rather, it is a bias that explains the emergence of a moral norm. The innate bias against incest prohibits sexual intercourse between family members and childhood associates, but does not in and of 
itself explain why all human groups tend to have moral norms prohibiting incest. However, the Westermarck mechanism may serve as an innate bias that favours the emergence of moral norms that prohibit incest. ${ }^{3}$

Recall that Haidt claims that when asked why incest is wrong despite positive consequences, individuals are dumbfounded in explaining why it is wrong. While Haidt, Dwyer, and Hauser claim that dumbfounding is a product of the moral faculty, there may be nonnativist, yet still evolutionary explanations for moral dumbfounding. For example, with regard to incest avoidance and the gut reaction that incest is wrong regardless of any positive benefits, we may simply have an innate tendency to avoid incest due to evolutionary factors, but not an innate moral rule against incest avoidance. Because incestuous relationships can and will cause a genetic depression, there is reason to believe that humans did in fact evolve with a tendency to avoid incest, and because this tendency was propagated throughout human evolution, it explains the quick and automatic judgment that incest is wrong even if there are no negative consequences, as well as explaining why we maintain that it is wrong, gross, or disgusting even if the act somehow produces positive consequences.

Sripada argues that humans evolved with certain innate biases, such as disgusted reactions to incest or murder, that guide moral decision-making. Innate biases that were propagated in humans, for nonmoral reasons, guide moral decision-making by being implicitly elicited when we are confronted with various moral situations. Sripada argues that the Innate Biases Model can adequately explain where moral norms come from and why individuals make specific moral judgments in certain situations. For example, we tend to avoid incest and judge incest as wrong and impermissible because humans evolved with an innate bias against it. Because incest causes a genetic depression, there is reason to speculate that incestuous 
relationships were condemned and perceived as impure, eventually creating a disgusted reaction to incest. This reaction may have been propagated in the form of an innate bias against incest, which is implicitly elicited when confronted with situations involving incestuous relationships. In Sripada's words, the Innate Biases Model argues that the widespread presence of certain kinds of moral norms across human groups may be explained in terms of the fact that the innate structure of the mind is predisposed to favour the emergence or propagation of these norms or predisposed to disfavour the emergence or propagation of other competing norms. However, unlike in the case of the Principles and Parameters Model...an innate bias does not require or preclude the presence of any particular moral norm or set of moral norms in the Norms Box. ${ }^{4}$

We may be disposed to have a disgusted reaction to incest because it reduces the fitness level of our kin, but this does not entail that we have an innate moral principle against incest. The innate bias of disgust is what leads to the judgment that incest is wrong. The innate bias of disgust that is elicited when thinking about incest leads most individuals to not only judge incest as wrong, but leads individuals to maintain that incest is wrong even if there are not any negative consequences following the action. We simply have an innate bias against such actions and will therefore always judge incest as wrong, regardless of the circumstances in which the action took place. The Innate Biases Model does not argue that the human brain comes equipped with a specific moral principle or a specific moral foundation prohibiting incest. Rather, certain biases that are present in the human mind, such as a bias of disgust when thinking of incest, will shape cultural norms and moral judgments.

The Innate Biases Model is more plausible than Haidt's view, the view that we condemn incest even if the consequences are positive because of innate foundations of morality, which is 
consistent with Haidt's conception of the role emotions play in moral decision-making. Rather than arguing in favour of innate universal moral domains, Sripada argues that biases shape the emergence and maintenance of moral norms. Recall that Sripada claims that we may be disposed to have a disgusted reaction toward specific actions such as incest because it reduces the fitness level of our kin, but this does not entail that we have an innate moral principle against incest or an innate foundation of purity guiding prohibitions against incest. Rather, the emotion-based bias accounts for the judgment that incest is wrong.

One way that innate biases work in spreading norms is through transmission. Innate biases allow norms to emerge and spread, where innate biases cause the reaction to a moral dilemma or situation. That being said, innate biases are not being transmitted, but the norms that emerge from innate biases are. One individual or a group of individuals can influence whether another group accepts or rejects the moral norm in question. For instance, Sripada notes that some moral norms may be accepted by a group of individuals because they are preferable to their psychology. Daniel Dennett also notes that memes, or ideas, of morality tend to spread and are maintained if they are catchy and useful to a group of individuals. Norms are also transmitted depending on which emotions are elicited, and how strong those emotions are. Sripada, alongside Prinz and Nichols, argues that if a norm is bound with a strong emotion, the norm will be maintained and exist in society longer than a norm that fails to implicate a strong emotion. Recall that Nichols points out that many contemporary etiquette norms are still around because they elicit disgust, and the norms that fail to implicate disgust are no longer part of contemporary etiquette. The same reasoning applies to harm norms. Nichols claims that "the fact that we are emotionally sensitive to others' suffering helps to explain why the harm norms ended up being so successful. For as harm norms entered the culture, their emotional resonance would have 
contributed to their cultural cachet." Thus, emotions and innate biases that are constituted by emotions play a significant role in determining which norms will be part of a society's morality.

Recall from Chapter I that Haidt argues in favour of moral modules that guide moral decision-making when looking at commonalities between cultures, and he gives the example of the cooties game in support of his theory. Haidt uses this example to show that innate moral modules develop with age; we begin to see the development of the purity domain or module when children begin playing the cooties game, where children give cooties to less attractive and less wealthy children, or children that should be deemed as impure. Haidt claims that despite negative reinforcement from educators and parents, children continue to play this game.

However, we do not need an innate moral module to explain the emergence or maintenance of the cooties game. Again, we can explain the cooties game by referring to innate biases, where norms spread if it accords with one's psychology, is catchy, easy to remember, etc. As Sripada notes, "some sorts of normative rules may be more or less 'attractive' due to the way they interact with one's preferences, aversions, emotions, and other elements of one's psychology...the transmission process will be influenced systematically by all of these factors." Thus, we do not need to postulate an innate purity foundation to explain why children play the cooties game.

In an attempt to explain new moral rules, Sripada suggests that new moral rules might emerge because humans face a novel social or ecological situation. For example, if there is a shortage of food or if there is a new technique for hunting game that requires cooperation, "a new moral norm may need to be proposed in order to deal with the problem of how the meat should be divided among the hunters." Also, a moral norm may emerge because the leader of a tribe or group proposes a new norm. For instance, if a powerful leader or prestigious individual 
has an aversion to eating pork, the individual can use their power to create a food taboo prohibiting consumption of pork. Consider the Spanish language and the rule of pronouncing the letter ' $\mathrm{J}$ ' as an ' $\mathrm{H}$ '. This rule was formed on the basis that a historical Spanish king was unable to pronounce the letter ' $\mathrm{J}$ ' and pronounced it as an ' $\mathrm{H}$ ' instead. In an attempt to save the king from embarrassment, the Spanish adopted the rule of pronouncing the letter ' $\mathrm{J}$ ' as an ' $\mathrm{H}$ '. We also tend to see similar trends in pop culture.

Continuing with the incest example used above, the Westermarck Mechanism works as an innate bias against incest and may have spread because of the genetic defects caused by incest and because it is attractive to one's psychology or cultural beliefs. This may also explain why the norm appears to be universal but differs in each culture. Recall that the Innate Biases Model argues that the brain is equipped with certain innate structures, such as aversions and preferences, and these biases serve to create and maintain moral norms, where some norms are chosen over others because they accord with a group of individuals' psychology or seem attractive to the beliefs of that group. The innate biases operate at a general level, where there is substantial variation on the content of the norm that is formed from the bias. For instance, depending on child rearing patterns in each culture, some cultures may consider children who are raised together but are not related to be immediate family members, where the Westermarck Mechanism will lead to the development of an aversion to sexual relationships with childhood friends. In some cultures, incest extends to stepbrothers and stepsisters and to first and second cousins, while in other cultures sexual relationships between cousins are permitted. Thus, we will witness a significant amount of cross-cultural difference in moral norms. As Sripada notes, innate biases such as the Westermarck aversion seldom determine in any precise or detailed way the content of the moral norms that they serve to favour. As a result, there 
will inevitably be a significant role for a number of other features, including the existing cultural context and, indeed, sheer happenstance, in determining the specific content of moral norms. ${ }^{8}$

Although this may seem like a PP component in the Innate Biases Model, Sripada claims that this variation across human groups is not best described in terms of parameterized variation, but in terms of what he calls thematic clustering. Thematic clustering is where "moral norms cluster under certain high-level themes with much residual variation at the level of specific content." One of the differences between thematic clustering and PP is that innate biases operate at a very general level, where "innate biases typically favour moral norms with certain broad thematic contents, but they leave open the potential for substantial variability with respect to the specific moral rule that is favoured." 10 The Innate Biases Model argues that there are numerous biases that are part of human psychology, and this is, of course, universal, as all human brains are similarly organized. These biases, the Westermarck aversion being one, operate at a general level and determine the content of the norm that emerges from the bias. For instance, if there is a familial structure in a community where maternal cousins are raised together, then the Westermarck mechanism will lead to the development of an aversion to having sexual relationships and procreating with maternal cousins. ${ }^{11}$ Although PP endorses a similar line of reasoning, the main difference between the two models is that PP requires exceptionless generalizations. The Innate Biases Model does not require exceptionless generalizations, while PP does, as the pattern of variation in moral norms across cultures is much too complex to be accounted for via rigid parameters. Moreover, the distributional pattern of moral norms, such as incest norms, exhibits thematic clustering, where most human groups have norms that fall under 
high-level themes, such as themes prohibiting incest, and the themes correspond to the content of the corresponding innate biases. ${ }^{12}$

At the level of specific content, where different cultures differ in their treatment of these high-level themes, moral norms differ significantly and exhibit quite a bit of variation. This line of reasoning applies to all norms. For example, harm prohibitions are a high-level theme that is caused by the innate bias of revulsion and repugnance against harming in-group members, but who counts as in-group members differs among cultures. Another high-level theme is meateating, where the bias of disgust when thinking of eating certain kinds of meat serve as meateating prohibitions. This theme varies across cultures and is dependent on whether the bias is elicited and to what extent, in addition to how it fits with the group's culture. For example, eating pork in Islamic cultures is prohibited because it is seen as impure and possibly disgusting, while eating most meats, especially beef, in Hindu cultures is perceived as immoral due to the belief in the transmigration of the soul. High-level themes are universal, while the specific content of high-level themes varies.

However, it is important to keep in mind that these prohibitions may be in place due to religious authority. Although innate biases do not play a role in following norms that are dependent on authority, giving innate biases a role in addition to accepting that authority plays a role in morality better explains the emergence of moral norms than moral nativism. Although both PP and thematic clustering appear similar in nature, thematic clustering more plausibly accounts for variation in moral norms across human groups, and the Innate Biases Model makes fewer commitments than moral nativism in explaining moral behaviour and variation in moral norms. 
This proposal explaining the emergence of norms and variation in norms across cultures appears more promising than PP. For instance, we do not need to appeal to innate principles in the human brain to account for moral acquisition. Rather, we can appeal to biological dispositions. Also, variation across cultures seem to work better with the Innate Biases Model than it does with PP; certain biases and biological endowments are shaped differently depending on one's culture, environment, and emotions. The Innate Biases Model also makes weaker commitments than moral nativism and can explain variation in moral norms more plausibly than moral nativism, thus we have reason to reject nativism as the best explanation for human morality.

Sripada's conception of morality better accounts for changes in moral norms and crosscultural variation than PP, which gives us further ground for dismissing moral nativism. First, $\mathrm{PP}$, as described in Chapters I and II, claims that the principles of morality, namely, the content of morality not just the capacity to moralize, are innate, while the Innate Biases Model argues that biases are innate, and does not need to incorporate innate moral principles to explain moral norms, moral acquisition, or changes in moral norms over time. I have discussed the problems with PP in Chapter II therefore I will not repeat them here. Instead, I wish to emphasize that, in an attempt to explain cross-cultural variation and changes in norms throughout history, the Innate Biases Model better accounts for these changes by appealing to the way certain norms fit with a group's psychology in addition to the fact that we tend to accept norms that we find attractive or beneficial.

In line with Prinz, Sripada also creates a place for emotions in his theory of moral acquisition, where these emotions serve as the foundation of biases and are considered biases as well. Without the emotion disgust, the Westermarck aversion may be non-existent. If we did not 
have an aversion against procreating with family members, we would not find it disgusting and may not feel prohibited from engaging in such an act; if we are not repulsed by the idea and if we do not find the consequences or the act of incest repulsive, disgusting, or impure, we probably would not feel compelled to follow incest prohibitions. According to Sripada's theory, without the bias of disgust and the Westermarck Mechanism, incest prohibitions would be non-existent. Sripada notes, like Nichols, that innate emotions play a significant role in maintaining moral norms as well as assisting in the emergence of moral norms. Essentially, innate emotions, such as sadness, and more complex emotions, such as sympathy, serve as the basis of harm norms, and the emergence of new or altered harm norms tend to enjoy an advantage in cultural evolution due to the fact that they accord well with certain aspects of human psychology. ${ }^{13}$ Again, it is not always the case that moral norms will require the basis of an emotion or innate biases, as religion and authority can account for many moral norms. However, in addition to accepting that some moral norms may not be based on affect and emotion, many, if not most, moral norms may be based on innate biases and negative affect.

The Innate Biases Model adequately explains the emergence, transmission, and maintenance of moral norms in addition to explaining changes in moral norms without facing the problems that PP faces. Not only does the Innate Biases Model provide a sound account of moral acquisition, but it does so without postulating a moral faculty, making fewer commitments than moral nativism, thereby giving us reason to reject moral nativism.

\section{Memes and Cultural Evolution}

Let us turn to memes and cultural evolution, where I draw on Dennett's work in an attempt to show that there are other plausible alternatives to moral nativism, alternatives that can be reasonably accepted. Dennett's theory of memes can be viewed either as a supportive theory 
to the other two nonnativist theories, or may be viewed as a separate theory, as memetics can also provide a plausible theory of moral norms and moral acquisition. Dennett's theory can be viewed as a supportive theory to a nonnativist theory such as the Innate Biases Model or Prinz's ECM to be discussed below, as memetics can work with these theories in providing further support. Alternatively, Dennett's theory can be viewed as a separate theory, where memetics, on its own, can account for the emergence, transmission, and maintenance of moral norms, where the ECM and Innate Biases Model are not needed to support memetics.

This section will focus on determining whether memes and cultural evolution are plausible explanations for the transmission and emergence of moral norms. I have two aims for this section. First, to supply Prinz and Sripada's theories with an additional component that further support their theories, thereby providing another reasonable alternative to moral nativism; and second, to show that memetics, on its own, can plausibly account for moral behaviour and moral norms. It is also important to point out that this account of morality does not dismiss the role of certain biases and emotions in moral decision-making, although the theory of memetics does not require that an individual have an innate bias or tendency in order for a meme to spread. Another factor that is important in discussing memetics is that while memetics can explain the emergence of moral norms, it is better equipped to explain the transmission and variation of moral norms, and that will be my primary focus here, although I will also touch on how memes can cause moral norms to emerge.

In Darwin's Dangerous Idea, Daniel Dennett reworks Dawkins' concept of the meme. A meme is simply an idea, which is defined by Dennett as "the name for an item of cultural evolution." ${ }^{\prime 4}$ Memes, like genes, depend on the ability to replicate and reproduce in order to ensure survival. Like genes, memes also need a host, more specifically, a human brain, in order 
to be transmitted. However, unlike genes, memes are transferred through the workability, acceptability, and creativity of an idea. Memes are ideas and do not need a biological function in order to ensure survival. Rather, the idea needs to be reproduced enough through means of cultural transmission in order to ensure survival. Memes are acceptable ideas that are catchy, valuable, and easy to memorize, which ensures the reproduction of the ideas. The fact that some memes may be counterintuitive to many individuals, such as the cooties game described by Haidt in Chapter II, does not entail that the meme will not spread.

"What we are is very much a matter of what culture has made us." 15 This is the base that I want to use to support the view that memes, not an innate set of principles, assist in moral decision-making. Let us take a look at how these two factors may account for the emergence, transmission and maintenance of moral norms. We have spent quite a bit of time discussing harm norms throughout this paper so I will be using harm norms as the primary example in this section. Moral nativists attribute cross-cultural differences to both the environment and a moral faculty. For instance, Hauser argues that harm norms differ in southern states and northern states of the USA because any innate and unconscious principle guiding harm norms are dependent upon how cultures treat harm, honour, etc. For Hauser, this is just another parametric variation on an innate principle. He argues that southerners are more aggressive than northerners because they have a longstanding history of defending honour in violent ways, and will therefore allow the permissibility of harm in order to defend one's honour. He claims that "in the South, not only are people more likely to respond aggressively to insult, but they expect others to respond violently to insult." ${ }^{, 16}$ A northerner would walk away from an insult because it is the proper thing to do, but if a southerner walked away he would be perceived as a wimp. The distinction between how the north and south treat violence and harm can also be found in city names. Cities 
in southern states (War, West Virginia and Gun Point, Florida) ${ }^{17}$ appear more aggressive than cities in northern states (Mount Joy, Pennsylvania and Peace Dale, Rhode Island).$^{18}$ Hauser provides compelling arguments to support the view that southerners are more aggressive than northerners and therefore treat harm prohibitions differently. Hauser is correct in drawing this conclusion but may be wrong in supposing that that harm prohibitions are part of an innate moral faculty and that parametric variation is what accounts for the differences.

A plausible alternative is that memes, the idea that you are wimpy if you do not respond violently to an insult or the idea that it is not proper to walk away from an insult, allow these norms to emerge and spread. When humans respond in certain manners to specific situations and are not condemned for their reactions, the reactions and related behaviour can spread as many individuals will assume that this is the correct or permissible way to act in similar situations. For a good analogy, look at pop culture and fashion trends; when an individual who is in highstanding and is well regarded acts a certain way or dresses in a particular manner, other individuals tend to act and dress in similar manners. Essentially, it becomes the norm until another individual in similar standing does something differently. Humans copy humans, especially when the behaviour appears advantageous.

There are also evolutionary considerations for how we respond to harm. For instance, we protect ourselves, our kin, and family members and harm those who disrupt the process. Dennett's conception of memes and cultural evolution does not deny the designed structure of the human brain; in other words, this theory does not contest capacity nativism. Designed structure can be defined as the innate structures in the brain that allow humans to moralize (not to be confused with innate principles, as designed structure is not the same as an innate principle), such as emotions and capacities (e.g., a theory of action). Designed structure does not 
have to entail moral principles due to the fact that not all humans can be said to have the innate moral principles that nativists argue in favour of. Rather, what is included in designed structure are the capacities that are present in all humans, such as the capacity to build boats, the capacity to think, the capacity to speak, etc. That being said, memes can account for harm prohibitions and cultural variation in the following way: we tend to protect ourselves from harm and the way in which we protect ourselves from harm differs from the way in which other individuals protect themselves from harm. It may be that copies and copies of the meme 'respond violently when you are insulted to protect your honour' persisted and multiplied in southern states because it accords with their citizens' psychology and their culture's long standing history, which explains the difference in harm prohibitions in northern and southern states. The fitness and survival of a meme depends on how many times it is replicated and how well it works. For instance, responding violently to an insult may work well for many southerners where an insult is harmful to one's honour, but may not work well for many northerners where the individual who is doing the insulting appears to be harming his reputation more than the insulted individual. This is true of all memes. Dennett notes that "memetics predicts conspiracy-theory memes will persist quite independently of their truth, and the meme for faith is apt to secure its own survival, and that of the religious memes that ride piggyback on it, in even the most rationalistic environments." 19 As long as a meme is catchy, useful, and replicated and reproduced enough it is bound to stick around and impact human behaviour.

This line of reasoning can be applied to all norms. For instance, the Machiguenga of Peru have different sharing norms because their idea of sharing works for them (e.g., the idea is catchy and useful) and has been replicated enough to stick around. Cultural evolution plays a significant role in morality, where ideas of morality are circulated and spread through cultural 
transmission. This also explains how new norms spread quickly; cultural evolution allow memes to spread quickly thus it will not be long before a new and catchy idea spreads and impacts human behaviour. In addition, a meme may be useful in one culture but may be irrelevant in another culture, which would explain variation in moral decision-making. For example, violating certain conventional norms is sometimes considered immoral in some cultures, such as eating meat in Hindu cultures. Because Hindus believe in the transmigration of the soul and are respectful of religious authority, meat prohibitions are moral norms in South Asian cultures but not our own. Another example that we have witnessed recently is social networking and the conception that important and popular individuals "tweet" (post their day-to-day transactions) their lives on the internet where this becomes a common form of keeping in touch with friends. This has significantly impacted human behaviour in North America, as many individuals want to be seen as significant and popular in the social circle, or they may have simply been influenced by this behaviour to believe that it is the norm to constantly update your life on the internet.

Dennett's account of memes can also explain why some moral norms change over time, as well as explaining variation across cultures and within cultures. For example, many children who are raised in a racist home tend to endorse racist principles that their parents indoctrinate in them. Another example we have seen throughout history is individuals who grow up in societies where their culture endorses notions of men being superior to women are likely to judge women as inferior, subject to domination, and dependent, and as a result, women are more than likely to internalize this notion of themselves as well. Or take, for instance, same-sex relationships, which were once thought to be impure and wrong on the basis of religion and societal norms. Currently, in North America, same-sex relationships, while not accepted by everyone, are now accepted by many individuals. Similarly, the incest taboo has changed over time and differs in judgment 
across cultures. For instance, if a society considers kin to extend to non-related childhood friends, engaging in procreation with a childhood friend may be seen as incest, while in some cultures incest may extend to first cousins, while some may limit incest to immediate family members. Similarly, incest norms have changed greatly in Western society as well. Up until the early nineteen hundreds, marriages between first cousins were not seen as morally reprehensible. In fact, even Darwin was married to his first cousin and acknowledged that the illnesses present in his children were a product of his marriage. ${ }^{20}$ Norms can change in a culture throughout history depending on how attractive the norm appears and is also determined by whether the norm accords with the group's psychology. For instance, incestuous relationships with first cousins probably became morally reprehensible in Western society when it became known that procreation between first cousins cause serious birth defects or when first cousins began living in households together. Although we are biologically built to resist incest, incest norms extending beyond immediate family may have been put into place at a later point in time after birth defects in children who were a product of cousin marriage became known. Cultural evolution works quickly, and we do not need hundreds of years to see changes in a norm. According to this account of morality, we do not need to postulate a moral faculty in order to explain the incest taboo. Rather, memes and cultural evolution, together with capacity nativism, are sufficient in explaining these norms.

Again, this view does not dismiss biological factors or emotions, but may work with these factors to explain morality. This is yet another theory that may or may not be correct. My goal here was to provide further support for a reasonable alternative to moral nativism that could be accepted as a plausible theory. Memetics plausibly accounts for the emergence, transmission, 
and maintenance of moral norms and makes weaker commitments than moral nativism, therefore, we have stronger reasons for endorsing nonnativism than nativism.

\section{Emotions Constitution Model (ECM)}

Finally, let us turn to the theory that moves the furthest away from nativism of the three theories presented in this chapter, a theory that can account for morality in a less complex manner than nativism, making weaker commitments than nativism. Prinz, like Nichols, defends a sentimentalist account of morality. Arguing in accord with eighteenth century Scottish philosopher David Hume, Prinz claims that "morality is a human construction that issues from our passions. ${ }^{.21}$ Although Hume tried to resist moral relativism, Prinz argues that his position leads to relativism due to the fact that on his theory, concepts such as good, bad, right, wrong, and ought are concepts that express sentiments, and these concepts are subjective. Without getting into too much metaethical theory, Prinz argues that when a person says an action is obligatory, they are expressing a prescriptive sentiment, where a prescriptive sentiment is a complex emotional disposition. For instance, if we think action $\mathrm{X}$ is obligatory, we feel that we should $\mathrm{X}$, and would feel guilty if we did not X, and would feel angry toward those who do not $X$. This is an instance of the internalization that was discussed in Chapter II. Essentially, if we feel that a specific action $\mathrm{X}$ is obligatory, we would feel motivated to $\mathrm{X}$.

Take the example of donating to Children's Fund. Some individuals do not believe that they have an obligation to donate money to this charity while others do. Those who do not believe that they have an obligation to donate normally will not feel guilty for not donating and will not feel anger toward those who do not donate. However, those who feel morally obligated to donate will probably feel guilty if they forget to mail their monthly donation and would probably be angry or outraged at someone who decided to unnecessarily spend a large sum of 
money on purchasing a car rather than donating to Children's Fund. Thus, "the good is that which we regard as good,"22 according to the ECM. Although it is not always the case that we feel motivated to act by actions that we consider obligatory, we are usually motivated to act or have good reasons for not acting.

If emotions constitute our moral judgments, as Prinz argues, we may witness variability in our moral judgments, such as making the claim that stealing is permissible under certain circumstances but forbidden in others. For example, in the well-known ethical dilemma of Heinz who steals from a pharmacy to save his wife's life, most individuals do not claim that Heinz is morally wrong for stealing from the pharmacy. Rather, on Prinz's account, they judge his action as permissible and even obligatory because the emotions associated with stealing are in competition with emotions of saving a life. Thus, according to the ECM the feelings of saving a life are stronger than those of stealing which is why most individuals may judge Heinz's action as morally permissible. No one will be harmed if Heinz steals from the pharmacy, but an individual will die if Heinz does not steal from the pharmacy. The ECM does a good job of accounting for our moral judgments and variability in moral judgments.

According to Prinz, "rightness and wrongness...are things we feel,"23 and this explains why we may judge some acts of stealing as morally permissible and others, or most acts of stealing, as impermissible. Rather than relying on innate principles represented in the mind to explain why we consider some acts of stealing permissible and others impermissible, it seems more plausible to credit the variability in our judgments to the role of emotions. This explanation not only accounts for the diversity of judgments between individuals in everyday moral decisionmaking, but also accounts for the variability in an individual's judgments. We do not judge actions solely from our moral principles or beliefs, but also on how we feel in a particular 
situation. Recall from Chapter II (Universality Does Not Require Innateness) that this accounts for the fact that we may find drinking apple juice out of a bedpan disgusting and possibly immoral (on Hauser's account it is a moral situation), but would hesitate to make the same judgment if we are desperately dehydrated in a desert. Our judgments are not universal across cultures and we find variability in our own judgments depending on the context. If morality were in fact innately represented, our moral judgments would not be context-dependent, and we have seen in Chapter II that our judgments differ depending on the context. Although on PP, some aspects of morality would be context-dependent while others wouldn't, it is difficult to differentiate when it would be appropriate to assume that morality is context-dependent. For instance, if we allow that all moral situations are context-dependent, we would no longer be endorsing a PP approach to morality; the rigid parameters would need to constrain variability in judgments. The ECM makes weaker commitments in an attempt to explain variability in moral judgments, and explains variability in a plausible manner.

A final component of the ECM that is significant to our discussion is how emotions, such as caring, can promote the formation of moral prohibitions, such as harm norms. Recall that Prinz claims that there may be universal constraints on stable societies, where stable societies promote and construct harm prohibitions. According to the ECM, simply because we do not have innate principles or an innate foundation prohibiting harm, it does not follow that humans living together in a society will be nasty or vicious toward one another. As Prinz states,

it must be noted that other species (e.g., squirrels, bird, and deer) don't go around killing each other constantly, but we are not tempted to say that they have moral rules against harm. They don't need such rules, because they have no biological predispositions to aggression against conspecifics. Likewise, we may have no such predispositions, so the 
lack of a biologically based prohibition against violence does not mean we are nasty and vicious. $^{24}$

Although the view that we have no predispositions to be aggressive or nasty toward one another can be contested, as we may have such predispositions, the point here is to emphasize that if the ECM can explain harm prohibitions and harm norms more adequately, or just as adequately, as moral nativism, there is reason to endorse nonnativism, as nonnativism makes weaker commitments than nativism. As seen in Chapter II (Universality Does Not Require Innateness), harm prohibitions are not universal and can be explained by appealing to the need for stability in society as well as positive regard toward in-group members. Where the nativist argues that innate moral principles are responsible for sharing norms and harm prohibitions, the nonnativist provides biological explanations and appeals to emotions to explain the emergence and maintenance of these norms, where the idea of a moral faculty can be easily dismissed, as there is nothing left for moral nativism to explain. Although nonnativism and nativism both have their fair share of unanswered questions and weaknesses, we must endorse the theory that makes weaker commitments, even if both theories adequately explain the data and observations presented.

Another explanation given by Prinz in an attempt to explain harm and reciprocity norms is that "we are innately gregarious: we socialize, form attachments, and value company. Rather than presuming that we are innately disposed to avoid harm, we might say we are innately disposed to take pleasure in other people's company." ${ }^{25}$ Although gregariousness is not a moral disposition, it may have implications for morality. For instance, we tend to help our loved ones and family members, and we dislike it when they are harmed. Caring is promoted by human friendship and love for family members, which promotes the emergence and formation of norms 
that prohibit harm. According to Prinz, harm norms may simply be by-products of the positive regard that we have for loved ones and family members. We do not need an innate harm principle, as Haidt claims, in order to account for harm prohibitions, sharing, caring about others, etc. Caring for others was selected for on the basis that on average, caring must directly or indirectly serve the fitness of those involved or it would have been selected against.

Here, it may appear as if Prinz endorses a form of the Innate Biases Model, arguing that we are predisposed with certain biases that form the emergence of norms. However, it is important to note that unlike Sripada, Prinz argues that emotions constitute our moral judgments, not innate biases, where gregariousness is a human predisposition, a feature that is common among humans, therefore it is inevitable that we will act in certain manners toward one another. This may cause the emergence of moral norms and may have implications for morality, where caring, friendship, and love for family members promote the emergence and formation of norms, but do not function as innate biases that cause moral norms to emerge. As Prinz notes, such norms may be by-products of our biological make-up. In simplistic terms, the ECM does not endorse the Innate Biases Model, but accepts capacity nativism, where innate emotions cause moral judgments and moral behaviour.

\section{Nativist Response}

Let us turn to how a nativist, such as Hauser, might respond to the ECM and the case for dismissing moral nativism. Recall from Chapter I that Hauser argues in favour of a moral faculty where individuals tend to act as Rawlsian creatures when making moral judgments. Although Hauser does recognize the importance of emotions in moral decision-making, he argues that 
emotions are not required to make a moral judgment, and if emotions are involved they are usually caused by a moral judgment rather than being a constituent component of it. On Hauser's account of morality, we tend to be dumbfounded when we make moral judgments due to the process of unconscious reasoning, and although it appears as if individuals have confidence in their judgments they are usually clueless when attempting to provide justifications. According to Hauser, this is how individuals normally make moral judgments and it explains their judgments in the Trolley Dilemma and Footbridge Dilemma. We judge the action of pulling the lever in the Trolley Dilemma as a permissible action and the action of pushing the large man off of the footbridge in the Footbridge Dilemma as an impermissible action because of our moral faculty. Embedded in our moral faculty is the doctrine of double effect, assisted by a theory of action, that allow us to make the judgment that it is permissible to pull the lever but impermissible to push the large an off of the footbridge. Similarly, Hauser argues that individuals respond in a universal manner to these types of dilemmas because of our moral faculty. Although Hauser admits that "in the absence of our emotional conductor (brain regions needed to activate moral emotions), there is little hope that moral behaviour will spring forth," ${ }^{26}$ he maintains that Rawlsian creatures do not need emotions to make moral decisions, "attending as they do to the causes and consequences of particular actions." 27

To support his argument, Hauser created alternatives to the trolley dilemmas that are similar to the classic dilemmas. In the first dilemma, there is a bystander named Ned who is taking a daily walk near the trolley tracks. Ned notices that the approaching trolley has lost control and is headed toward five hikers walking on the track. The banks of the tracks are so steep that the hikers will not be able to get off of the track in time. Ned is standing next to a switch that he can throw, and throwing this switch will temporarily turn the trolley onto a side 
track. There is a heavy object on the side track. If Ned throws the switch the trolley will hit the object and slow down, giving the hikers time to get off of the track. However, the heavy object is a large person. Should Ned throw the switch? ${ }^{28}$

In the second dilemma, there is a bystander named Oscar who is taking his daily walk near the trolley tracks when he notices that the approaching trolley has lost control. Again, there are five hikers on the track and the banks are too steep and the five hikers will not be able to get off in time. Oscar is standing next to a switch that he can throw, and throwing the switch will temporarily turn the trolley onto a side track where a heavy object is placed. If the trolley hits the heavy object, which is a large boulder, the trolley will slow down giving the hikers time to get off of the tracks. However, there is a person standing on the side of the track in front of the boulder, and this person will be hit and killed by the boulder if Oscar throws the switch. Should Oscar throw the switch? $?^{29}$

Hauser argues that individuals would make the judgment that it is not permissible for Ned to throw the switch but permissible for Oscar to throw the switch because if Ned throws the switch, he is intentionally committing harm and the consequences are also foreseen, where Oscar is causing harm but only as a foreseen side effect, not as an intended consequence. Hauser claims that we would have these intuitions about these dilemmas because of the doctrine of double effect, a principle that is innately represented in the human brain. ${ }^{30}$ For Hauser, "it is possible that our judgments reflect intuition, percolating up from unconscious and inaccessible principles of action." ${ }^{31}$ Similarly, recall that Mikhail argues, as difficult as it is to accept, that such concepts are innately represented in the mind and guide our moral judgments. This leads Mikhail and Hauser to conclude that we cannot be Humean creatures, where Humean creatures are guided by emotions and sentiments, because the Ned and Oscar dilemmas both entailed an impersonal 
action with similar consequences. If we are guided by emotions and gut reactions, impersonal acts would trigger similar responses. Yet this is not the case, as Hauser tries to show, and this leads Hauser to conclude that we are Rawlsian creatures "equipped with a mental barometer that distinguishes between killing as a means and killing as an unintended but foreseen side effect. ${ }^{\$ 32}$ What is more, innate moral principles, such as the doctrine of double effect, operate unconsciously but directly influence our judgments. In the same way that we know two solid objects cannot occupy the same space at the same time without taking a course in physics, Hauser claims that "much of our knowledge of morality is similarly intuitive, based on unconscious and inaccessible principles for guiding judgments of permissibility.,33

However, the nonnativist may respond that most individuals think it is impermissible to push the large man off of the footbridge but permissible to pull the lever because emotions are activated and elicited to a higher degree in the Footbridge Dilemma, which causes the judgment that killing is worse than saving lives. In the latter dilemma, we may elicit fewer emotions because it is an impersonal situation (e.g., we are not physically harming an individual), thus most individuals judge that saving five lives is better than taking one life. It is not the case that we do not activate any emotions when we make a judgment in the Trolley Dilemma. Rather, emotions may be elicited to a lesser degree because we are not physically harming an individual as we are hypothetically asked to imagine in the Footbridge Dilemma. With regard to Hauser's alternative trolley dilemmas, my hunch is that we would activate the brain region associated with emotions in Ned's dilemma to a greater degree than we would in Oscar's dilemma. If the variability in judgments can be explained by a nonnativist account, we have reason to endorse nonnativism rather than nativism, especially if it explains the data and observations by making 
fewer commitments than nativism. If the ECM can explain the acquisition of moral behaviour, then there is no need for innate moral principles.

Therefore, nonnativism can explain our moral intuitions and judgments about killing and letting die, and does so just as adequately as moral nativism and makes fewer commitments than nativism. Simply, we may elicit stronger negative emotions about killing than we do about letting die. As Prinz notes, if our action, such as pulling a lever to redirect the trolley, allows someone to die, this was not our primary intention, and we are able to focus our attention away from the victim and on our primary intention. When we focus on taking a life or physically harming an innocent person, we cannot focus our attention away from the victim because harming the victim is a primary intention. This may explain why the negative feelings brought about in the Footbridge Dilemma are much stronger than they are in the Trolley Dilemma; killing seems worse than letting die as a result of these stronger emotions. ${ }^{34}$ Contra Hauser, emotions may not be caused by moral judgments. Rather, emotions may be constituent components of moral judgments. Killing is more emotionally charged than letting die and these emotions are not caused by the judgment but are components of the judgment. Diminishing the emotional sensitivity toward killing by changing the scenarios in the Trolley Dilemma changes the judgment of permissibility in killing, which is sufficient in explaining variability in judgments. The less that emotions are elicited, the more permissible it is to take one life for five. On the causal influence account, such as Hauser's, there should be cases where individuals moralize without invoking emotions, and there should not be cases where emotions are the basis for a moral judgment, as seems to be the case in the Trolley Dilemma, Footbridge Dilemma, and the alternative trolley dilemmas (the Ned and Oscar dilemmas). Therefore, we do not need nativism to explain the variability in judgments in the trolley dilemmas. Again, if there is an 
emotional reaction to the dilemmas that explain the moral judgments, then there is no need to postulate innate moral principles.

Recall from Chapters I and II that on Mikhail's account of moral acquisition, young children calibrate the level of punishment they assign to harmful acts based on intent, provocation, fairness, etc. According to Mikhail, children are able to make these judgments because of an innate moral faculty and children are not exposed to any stimuli that allow them to understand that acts that produce more harm are treated more seriously and are punished more seriously than acts that produce only a minimal amount of harm. However, we do not need to postulate an innate moral faculty to account for this ability. Rather, we can endorse the theory that makes fewer commitments, where moral judgments are often accompanied by emotions, and the stronger the emotions, the stronger the punishment. For instance, the ECM argues that "crimes against children are often deemed worse than crimes against adults and they also seem to stir up stronger emotional responses." 35 As previously noted with the trolley dilemmas, we may judge the trolley dilemmas differently depending on the intensity of the dilemma; the degree of impermissibility or permissibility corresponds with the degree of physical contact and harm involved. Thus, we may tend to think that killing is worse than letting die because it arouses stronger negative emotions, and the same line of reasoning can be applied to most moral judgments. This is enough to explain why we witness cross-cultural differences and may also explain why we witness variability in our own moral judgments.

In this section, we have seen that on the ECM, emotions are constituent components of moral judgments, where environmental factors, education, and the need for stable societies play a role in moral acquisition and are more plausible accounts of moral decision-making and the emergence of moral norms than moral nativism. Although these factors are consistent with moral 
nativism, they weaken the moral nativist position simply because we do not need to postulate an innate moral faculty if emotions, culture, and learning can account for moral acquisition on its own.

\section{Conclusion}

Throughout the course of this chapter I have attempted to provide plausible and reasonable alternatives to moral nativism, alternatives that make weaker commitments yet explain the acquisition of moral behaviour in a more plausible, or just as plausible, manner than moral nativism. Returning to Chomsky's quote at the beginning of this chapter, we should only accept a nativist theory, such as that of the language faculty, if there are no plausible alternatives. Because language is expressed by children at an extremely young age with very little assistance from the environment, it is reasonable to accept a nativist theory about language. Morality may be displayed by many children at a relatively young age, however, the environment provides sufficient stimuli for children to acquire moral behaviour and norms. Moral nativism should be accepted only if no other account of moral acquisition is plausible or reasonable. As I have shown throughout this chapter and in Chapter II, we have grounds for rejecting moral nativism and endorsing a nonnativist account of morality, an account that makes fewer commitments than moral nativism. A nonnativist theory of morality, whether it is the ECM, the Innate Biases Model, or memetics, reasonably accounts for moral acquisition, the emergence and transmission of moral norms, and the maintenance of moral norms. Again, the topic of whether nonnativism answers questions more plausibly than nativism can be contested. However, what I have attempted to show in this chapter is that even if both theories can account for the acquisition of 
moral behaviour, nonnativism makes weaker commitments and is able to explain moral acquisition just as plausibly, if not better, than moral nativism. What is more, nonnativism does not make the additional commitments that nativism requires, therefore we have an additional reason for accepting nonnativism. Thus, there are no grounds for accepting moral nativism.

\section{Notes}

${ }^{1}$ Noam Chomsky, Rules and Representations (New York: Columbia University Press, 1980), 86-87.

${ }^{2}$ Nichols, 27.

${ }^{3}$ Sripada, "Nativism and Moral Psychology", 355.

${ }^{4}$ Chandra Sekhar Sripada, "Nativism and Moral Psychology: Three Models of the Innate Structure That Shapes the Contents of Moral Norms" as in article in Walter Sinnott-Armstrong, Moral Psychology: The Evolution of Morality: Adaptations and Innateness vol. 1 (Cambridge: MIT Press, 2008), 333.

${ }^{5}$ Sripada, 333.

${ }^{6}$ Sripada, 336.

${ }^{7}$ Thank you to Dr. Andrew Brook for providing me with this example.

${ }^{8}$ Sripada, 338.

${ }^{9}$ Ibid.

${ }^{10}$ Sripada, 337.

${ }^{11}$ Ibid.

${ }^{12}$ Sripada, 338.

${ }^{13}$ For more on this view, see Sripada 2008 and Nichols 2004.

${ }^{14}$ Daniel Dennett, Darwin's Dangerous Idea: Evolution and the Meanings of Life (New York: Simon and Schuster, 1995), 342.

${ }^{15}$ Dennett, 340.

${ }^{16}$ Hauser, 135.

${ }^{17}$ Hauser, 134.

${ }^{18}$ Ibid.

${ }^{19}$ Dennett, 349.

${ }^{20}$ See Jonathan Clements, Darwin's Notebook, 2009.

${ }^{21}$ Jesse Prinz, The Emotional Construction of Morals, (New York: Oxford University Press, 2007), 2.

${ }^{22}$ Prinz, 1.

${ }^{23}$ Prinz, 13.

${ }^{24}$ Ibid.

${ }^{25}$ Prinz, "Is Morality Innate?", 375.

${ }^{26}$ Hauser, Moral Minds, 229 (parenthesis mine).

${ }^{27}$ Hauser, 231.

${ }^{28}$ Hauser, 117, 118.

${ }^{29}$ Hauser, 118.

${ }^{30}$ Hauser, 118-122.

${ }^{31}$ Hauser, 121.

${ }^{32}$ Hauser, 125.

${ }^{33}$ Ibid.

${ }^{34}$ Prinz, 23.

${ }^{35}$ Prinz, 21. 


\section{Conclusion}

In this paper, I have attempted to show that moral nativism does not plausibly account for the acquisition of moral behaviour and moral norms. In particular, I have shown that nonnativism is more plausible, and makes weaker commitments, than moral nativism.

In Chapter I, we explored the moral nativist theories presented by Hauser, Dwyer, and Haidt, and looked at the poverty of moral stimulus argument, moral dumbfounding, PP, and the argument from universality.

In Chapter II, I critically examined moral nativism and the main components of the nativist theories presented in Chapter I. In particular, I showed that our stimuli are not impoverished with regard to moral acquisition, although it may be impoverished with regard to language acquisition. I have shown that dumbfounding is not a real phenomenon, and is unlike the dumbfounding that we experience in language. I have also argued that the LA is not a valid analogy, and language acquisition is significantly different from moral acquisition. I have also shown that it is problematic to make a jump from universality to innateness, and that universality in moral decision-making does not require innateness.

In the final chapter, Chapter III, I presented and analyzed three nonnativist theories, the Innate Biases Model, Dennett's theory of memetics, and the ECM, and argued that nonnativism makes weaker commitments than moral nativism and plausibly accounts for moral acquisition.

My intent for future research is to look at how the discipline of ethics may be effected by endorsing nonnativism, in addition to looking at the influence of nonnativism in metaethical theory. Some questions I hope to provide answers to are the following: what can we say about morality with regard to research in neuroscience that looks at the process of moral decisionmaking? What are the implications of a nonnativist view in ethical theory? Will a nonnativist 
theory lead us to dismiss current ethical positions? Does a nonnativist view lead to ethical naturalism or relativism about morality? As the field of moral psychology continues to prosper, we must look to how the disciplines of ethics and philosophy react to the arguments and data being presented. 


\section{Bibliography}

Anderson, Steven W, Antoine Bechara, Hanna Damasio, Daniel Tranel and Antonio R. Damasio. "Impairment of Social and Moral Behavior Related to Early Damage in Human Prefrontal Cortex." Nature Neuroscience 2, no. 11 (1999): 1032-1037.

Bechara, Antoine, Hanna Damasio, and Antonio R. Damasio. "Emotion, Decision Making and the Orbitofrontal Cortex. "Cerebral Cortex 10 (2000): 295-307.

Bechara, Antoine, Antonio R. Damasio, Hanna Damasio, and Steven W. Anderson.

"Insensitivity to Future Consequences Following Damage to Human Prefrontal Cortex." Cognition 50 (1994): 7-15.

Bolender, John. "The Genealogy of the Moral Modules." Minds and Machines 13 (2003): 233255.

Brook, Andrew and Robert J. Stainton. Knowledge and Mind: A Philosophical Introduction. Cambridge: MIT Press, 2000.

Broom Donald. The Evolution of Morality and Religion. Cambridge: Cambridge University Press, 2003.

Carruthers, Peter. "The Case for Massively Modular Models of Mind." In Contemporary

Debates in Cognitive Science, edited by Robert J. Stainton, 3-21. Malden: Blackwell Publishing, 2006.

Chomsky, Noam. Language and Problems of Knowledge: The Managua Lectures. Cambridge: MIT Press, 1988.

— Rules and Representations. New York: Columbia University Press, 1980.

Churchland, Patricia. Braintrust. New Jersey: Princeton University Press, 2011.

Clements, Jonathan. Darwin's Notebook: The Life, Times, and Discoveries of Charles Robert Darwin. New York: Running Press Book Publishes, 2009.

Dennett, Daniel C. Darwin's Dangerous Idea: Evolution and the Meanings of Life. New York: Simon and Schuster, 1995.

Dwyer, Susan. "How Not to Argue That Morality Isn't Innate: Comments on Prinz." In Moral Psychology: The Evolution of Morality: Adaptations and Innateness vol. 1, edited by Walter Sinnott-Armstrong, 407-418. Cambridge: MIT Press, 2008.

"Moral Dumbfounding and the Linguistic Analogy: Methodological Implications for the Study of Moral Judgment." Mind and Language 24, no.3 (2009): 274-296.

Dwyer, Susan and Marc Hauser. "Dupoux and Jacob's Moral Instincts: Throwing Out the Baby, Bathwater and the Bathtub" Trends in Cognitive Science 12, no.1 (2007): 1-2.

Fodor, Jerry A. The Mind Doesn't Work That Way. Cambridge: MIT Press, 2000.

- The Modularity of Mind. Cambridge: MIT Press, 1983.

Greene, Joshua, and Jonathan Haidt. "How (and where) Does Moral Judgment Work?." TRENDS in Cognitive Sciences 6, no. 12 (2002): 517-523. 
Greene, Joshua D., Leigh E. Nystrom, Andrew D. Engell, John M. Darley, and Jonathan D. Cohen. "The Neural Bases of Cognitive Conflict and Control in Moral Judgment." Neuron 44, (2004): 389-400.

Haidt, Jonathan. "The Emotional Dog and its Rational Tail: A Social Intuitionist Approach to Moral Judgment." Psychological Review 108 (2001).

Haidt, Jonathan and Fredrik Bjorklund. "Social Intuitionists Answer Six Questions about Moral Psychology." In Moral Psychology: The Cognitive Science of Morality: Intuition and Diversity vol. 2, edited by Walter Sinnott-Armstrong, 181-217. Cambridge: MIT Press, 2008.

Haidt, Jonathan, and Jesse Graham. "When Morality Opposes Justice: Conservatives Have Moral Intuitions That Liberals May Not Recognize." Social Justice Research (February 2006).

Harman, Gilbert. "Using a Linguistic Analogy to Study Morality." In Moral Psychology: The Evolution of Morality: Adaptations and Innateness vol. 1, edited by Walter Sinnott-Armstrong, 345-351. Cambridge: MIT Press, 2008.

Hauser, Marc. Moral Minds: How Nature Designed Our Universal Sense of Right and Wrong. New York: HarperCollins, 2006.

Hauser, Marc D., Liane Young, and Fiery Cushman. "Reviving Rawls's Linguistic Analogy: Operative Principles and the Causal Structure of Moral Actions." In Moral Psychology: The Cognitive Science of Morality: Intuition and Diversity vol. 2, edited by Walter SinnottArmstrong, 107-143. Cambridge: MIT Press, 2008.

Joyce, Richard. The Evolution of Morality. Cambridge: MIT Press, 2006.

Mikhail, John. "The Poverty of the Moral Stimulus". In Moral Psychology: The Evolution of Morality: Adaptations and Innateness vol. 1, edited by Walter Sinnott-Armstrong, 353-359. Cambridge: MIT Press, 2008.

Nichols, Shaun. Sentimental Rules: On the Natural Foundations of Moral Judgment. New York: Oxford University Press, 2004.

Pinker, Steven. "So How Does the Mind Work?" Mind and Language 20, no. 1 (2005): 1-24.

The Language Instinct. New York: Harper Collins, 1994.

"The Moral Instinct." New York Times, January 13, 2008.

Prinz, Jesse. Gut Reactions. New York: Oxford University Press, 2004.

"Is Morality Innate?." In Moral Psychology: The Evolution of Morality: Adaptations and

Innateness vol. 1, edited by Walter Sinnott-Armstrong, 367-406. Cambridge: MIT Press, 2008.

"Is the Mind Really Modular?." In Contemporary Debates in Cognitive Science, edited by Robert J. Stainton, 22-36. Malden: Blackwell Publishing, 2006.

"Reply to Dwyer and Tiberius." In Moral Psychology: The Evolution of Morality:

Adaptations and Innateness vol. 1, edited by Walter Sinnott-Armstrong, 427-439. Cambridge: MIT Press, 2008.

The Emotional Construction of Morals. New York: Oxford University Press, 2007.

Rawls, John. A Theory of Justice. Cambridge: Belknap Press, 1971, 1999. 
Samuels, Richard. "Is the Human Mind Massively Modular?." In Contemporary Debates in Cognitive Science, edited by Robert J. Stainton, 37-56. Malden: Blackwell Publishing, 2006.

Sripada, Chandra S. "Nativism and Moral Psychology." In Moral Psychology: The Evolution of Morality: Adaptations and Innateness vol. 1, edited by Walter Sinnott-Armstrong 319-343. Cambridge: MIT Press, 2008.

- "Reply to Harman and Mikhail." In Moral Psychology: The Evolution of Morality: Adaptations and Innateness vol. 1, edited by Walter Sinnott-Armstrong 361-365. Cambridge: MIT Press, 2008. 